\title{
Endogeneidad de los rendimientos educativos en México
}

\section{Edna María Villarreal Peralta*}

Perfiles Latinoamericanos, 26(51)

2018 | pp. 265-299

DOI: $10.18504 / \mathrm{pl} 2651-011-2018$

\begin{abstract}
Resumen
Los rendimientos educativos consisten en los incrementos salariales de los trabajadores que derivan del aumento en sus años de escolaridad. En el presente trabajo estimamos la evolución en México de tales rendimientos para el lapso 1995-2012 empleando tres métodos distintos. De ello se ha obtenido que en promedio un ańo de escolaridad hace crecer en $9 \%$ el salario de los trabajadores para el periodo de estudio. Asimismo, que dichos rendimientos aumentan ante más años de escolaridad, que las mujeres son las que han visto su mayor incremento, y que han descendido durante el periodo analizado.
\end{abstract}

\begin{abstract}
The returns to education consist of wage increases for workers due to the raise in their years of schooling. In this paper we estimate the evolution of these in Mexico from 1995 to 2012 using three different methods. Our results show in average that an increase of one year of schooling raises wages by $9 \%$ during the study period. We also find that these returns increase with years of schooling, and women get the higher returns, highlighting finally that there is a decline in these during the period analyzed.
\end{abstract}

Palabras clave: rendimientos educativos, salarios, endogeneidad, capital humano, México. Keywords: Educational performance, salaries, endogeneity, human capital, Mexico.

* Doctora en Economía Aplicada por la Universidad Autónoma de Barcelona, Departamento de Economía de la Universidad de Sonora | edna.villarreal@unison.mx 


\section{Introducción}

E

estudio del efecto de la inversión en educación sobre el crecimiento económico fue desarrollado a principios de 1960. Schultz (1961) afirmaba entonces que el rendimiento al invertir en capital humano se reflejaba en el incremento del ingreso que perciben los trabajadores que hubieren erogado en su educación. Schultz (1961), respaldado por Mincer (1962), Hansen (1963), Becker (1962,1964) y Hanoch (1967), integró un enfoque empírico cuya hipótesis central plantea que la formación de los trabajadores incrementa su productividad la cual estimula el crecimiento económico. Del mismo modo, todos estos autores aseveran que la rentabilidad de la inversión en educación es más alta que la del capital físico. A partir de estos trabajos se llevaron a cabo distintas investigaciones retomando el modelo de Mincer (1974) que se basaba en una función de ingresos que incluye variables de salarios, escolaridad y experiencia. Estos trabajos demostraron que, en diferentes países y periodos, y utilizando diversas técnicas, el ingreso está determinado por el nivel educativo de los individuos (McMahon, 1991; Card \& Krueger, 1992; Altonji, 1993; Ashenfelter \& Krueger, 1994; Cohn \& Hughes, 1994; Harmon \& Walker, 2001; Salas, 2002, 2004). Para el caso específico de México, los estudios sobre los rendimientos de la educación son numerosos y se ciñen a los planteamientos teóricos internacionales.

La elección del caso mexicano y del periodo 1995-2012 es en particular relevante por sus notables cambios. Entre estos destacan reformas económicas, institucionales, demográficas, la apertura externa y el cambio tecnológico intensivo en conocimiento. Además de las reformas en el sector educativo, como su desregulación ${ }^{1}$ en 1993 y el decreto de la obligatoriedad de la educación preescolar y secundaria en la misma fecha y de la media superior en 2013, entre otros (figura 1).

La obligatoriedad de la educación secundaria incrementó el gasto en educación básica en términos reales en 112\% entre 1990 y 1994 (gráfica 1). Asimismo, se observó un aumento de $40 \%$ en la escolaridad promedio ${ }^{2}$ de la población entre el ciclo escolar 1990-1991 (6.4 años) y el 2014-2015 (9.1 años) (gráfica 2). Sin embargo, ha tomado veinte años lograr la universalidad en la cobertura de la educación básica (3 a 14 ańos de edad), y apenas fue en el ańo 2000 que se incrementaron las tasas de matrícula en la educación universitaria en México (gráfica 3).

1 El gobierno central, mediante la Secretaría de Educación Pública (SEP), determina el monto de la transferencia federal a cada estado. Esta desregulación motivó la reorganización del sistema educativo en el financiamiento y en la distribución del gasto.

2 Históricamente es el indicador más utilizado para medir el capital humano en términos agregados. 
Figura 1. Principales reformas de la educación en México, 1993-2013

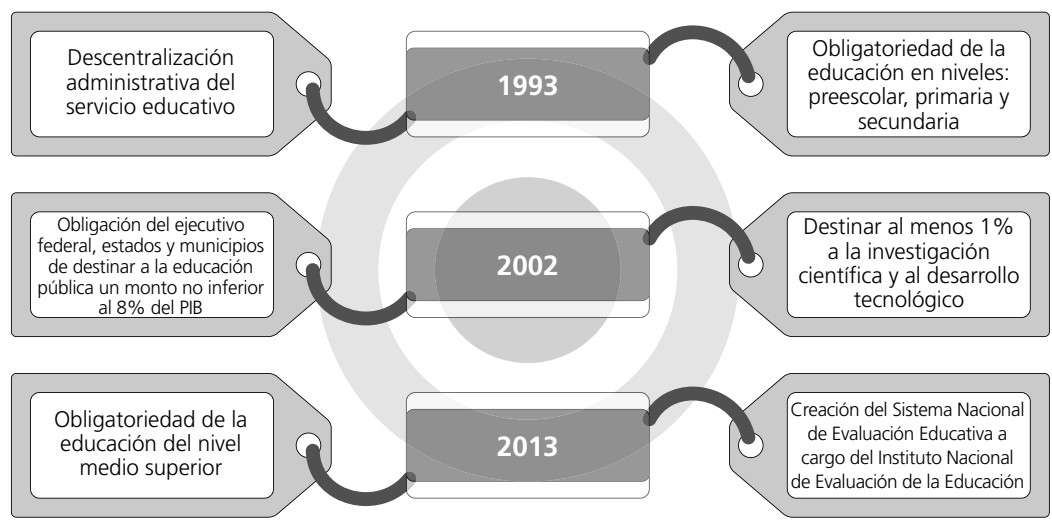

Fuente: CAIINNO (2016).

Gráfica 1. Variación en términos reales del gasto federal por nivel educativo

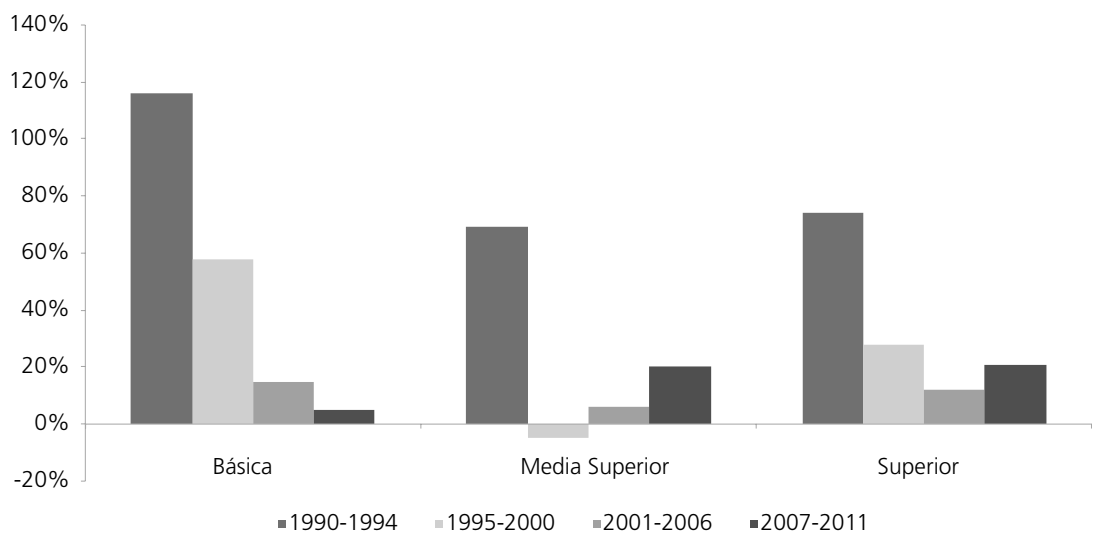

Fuente: Elaboración propia con datos del Panorama del Gasto Educativo en México (2012).

Gráfica 2. Años de escolaridad promedio, 1980-2017

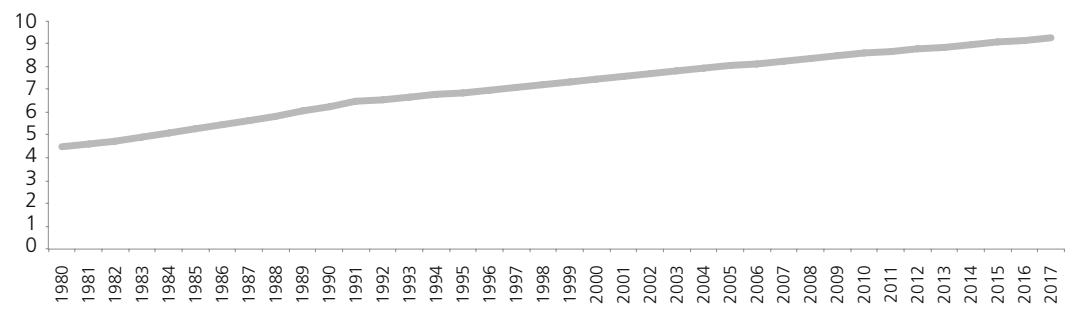

Fuente: Elaboración propia con datos del Reporte de Indicadores Educativos (2017) de la Dirección General de Planeación y Estadística Educativa de la Secretaría de Educación Pública (SEP). 


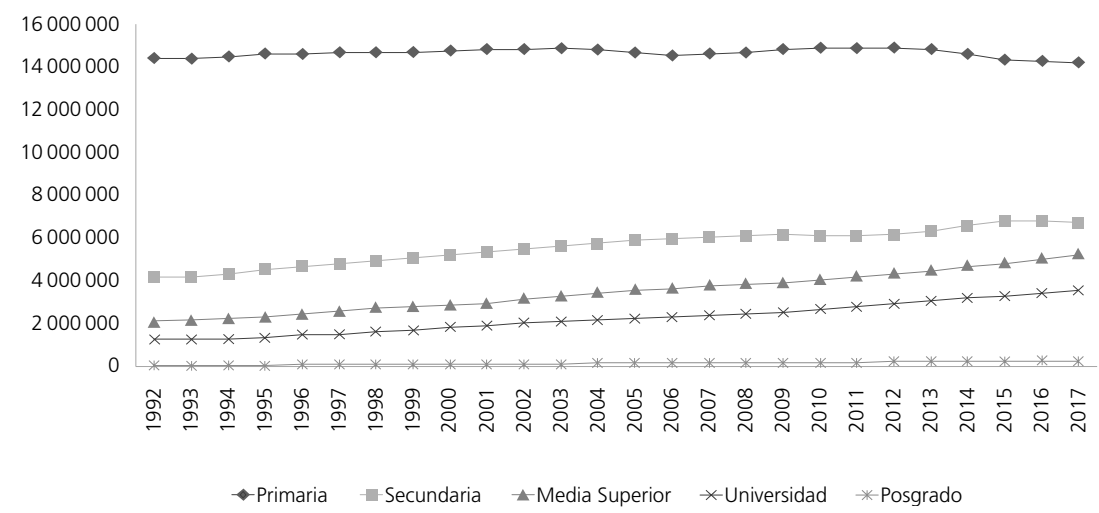

Fuente: Elaboración propia con datos del Reporte de Indicadores Educativos (2017) de la Dirección General de Planeación y Estadística Educativa de la Secretaría de Educación Pública (SEP).

Este trabajo se interesa en el estudio de la evolución histórica de los rendimientos educativos para México. La intención es evaluar el impacto del nivel educativo sobre los ingresos de los trabajadores mexicanos en el periodo 1995-2017. Para esto se ha emprendido una revisión de la evolución histórica de los rendimientos educativos en México calculándolos mediante tres estimaciones: 1) la de una ecuación minceriana tradicional que mide el incremento salarial del trabajador por un aumento de un año de escolaridad; 2) con la metodología de variables instrumentales se ha controlado la endogeneidad de la variable escolaridad a través del cambio legislativo sobre los años de escolaridad obligatoria, y 3) por niveles educativos se ha medido la prima salarial de su avance.

La evidencia empírica para México, aunque es amplia y responde a los planteamientos teóricos, contiene pocos trabajos que hayan considerado la posible endogeneidad del nivel educativo de los individuos, sobre todo por las limitaciones de información (Sariñana, 2002; Barceinas, 2003). Con estos argumentos, este trabajo se propone ahondar en el tema para complementar aportando estimaciones sobre la evolución de los rendimientos educativos.

El artículo se estructura como sigue: después de esta introducción, en la segunda sección se expone una revisión teórica; en la tercera, se resume la evidencia empírica del caso mexicano; en la cuarta se detalla la metodología empleada; en la quinta se describe la base de datos utilizada; en la sexta se presentan los resultados de las estimaciones econométricas y, finalmente, se añaden unas conclusiones. 


\section{Revisión teórica}

Los clásicos Cantillón (1755) y Smith (1776) afirmaron que el rendimiento de los factores de producción se explicaba por el progreso tecnológico y por el efecto de la educación sobre la acumulación de capital físico y humano. Con estos referentes, Schultz (1961), Becker $(1962,1964)$ y Mincer (1974) construyeron la base de la teoría económica que explica la influencia de la educación —en su sentido de variable de capital humano- sobre la productividad y el crecimiento económico. Estos trabajos concentran su hipótesis en la relación directa entre educación, crecimiento económico, ingresos y desarrollo, y sus autores fundaron la teoría del capital humano (TCH) afirmando que la educación debe ser interpretada como una inversión que los individuos realizan para aumentar su dotación de capital humano incrementando su productividad, la cual se reflejará en un mayor crecimiento de las economías.

Schultz (1961) explica las diferencias de productividad y salarios de los trabajadores para determinar la parte del crecimiento económico que no se explicaba por los factores tradicionales incluidos en la función de producción. Otro de los aportes de este autor es haber utilizado el gasto en educación como variable de aproximación de la inversión en capital humano, con lo que propuso el cálculo de la inversión humana a través del rendimiento y no del coste. El rendimiento de la inversión en capital humano se expresa como aumento del ingreso de los trabajadores que hayan invertido en educación. Schultz (1961) analizó en términos empíricos la relación entre crecimiento y educación para Estados Unidos durante el periodo 1920-1957 y sus resultados mostraron que el incremento de la educación explicaba entre el 16 y el $20 \%$ del crecimiento del ingreso nacional. Su conclusión más relevante fue que la formación educativa y los adelantos en conocimiento eran determinantes para el crecimiento económico.

Becker (1964), por su parte, se propuso determinar el efecto de la inversión en capital humano sobre el desempleo, el ingreso y el crecimiento económico. En su teoría, él conceptualiza el instrumento metodológico central de la TCH analizando los diferentes rendimientos de la inversión educativa. Para ello define el tipo de rendimiento privado y el social estudiando el coste-beneficio de la educación. Su trabajo lo lleva a delimitar distintos modos de invertir en capital humano, entre estos la formación laboral y la educación formal en las escuelas, mismas que permiten la acumulación de capital humano.

El modelo que Mincer (1974) formuló dentro de la TCH evaluó por primera vez la relación entre distribución salarial y capital humano. Fue así que, además de llevar a cabo un análisis empírico, configuró un marco conceptual y teórico del capital humano agregando la noción de tasa de rentabilidad de la 
educación. Mincer supuso que un individuo decide seguir estudiando en lugar de incorporarse al mercado laboral porque espera que así mejoren sus percepciones en dinero. Dicho autor señala que la experiencia juega un rol importante en los niveles de ingresos para los trabajadores.

Para la década de los setenta surgieron las teorías de la señalización, de la competencia de los puestos de trabajo y la radical que, paralelas a la тCH, también se ocupaban de la relación entre educación y empleo.

La teoría de la señalización, selección o del filtro fue impulsada por Arrow (1973), Spence (1973) y Stiglitz (1975). Con su planteamiento, esta teoría abrió la discusión acerca de si el aumento de los salarios de los individuos más educados proviene realmente de que la educación eleva su productividad, como lo afirmaba la тсн. Los postulados de la señalización argumentan que la educación no incrementa la productividad, sino que es una señal que sirve para identificar o seleccionar a los trabajadores con mayor capacidad o habilidad innata, algo útil para los empleadores.

Tanto la teoría de la señalización como la TCH coinciden en que un incremento de la escolaridad redunda en mayores ingresos, ya sea vía productividad o como señal enviada a los empleadores. Las diferencias entre ambas hipótesis radican en sus enfoques sobre las implicaciones para la productividad de los individuos y los costes sociales de la educación. Si fuera válida la postura de la señalización, el beneficio social de la inversión educativa sería nulo, ya que no aumentaría la productividad del trabajo. En este escenario, la inversión en educación solo serviría para mostrar a los empresarios la disciplina, inteligencia o motivación de los trabajadores, pero no contribuiría al afianzamiento de sus habilidades para ser más productivos. Así, el hecho de que los individuos más educados perciban mayores salarios representaría una búsqueda de rentas sin beneficios para la economía. Es decir, que los recursos asignados a la educación no traerían beneficios desde el punto de vista social, porque no se traducirían en un mayor stock de capital humano y por tanto no se alteraría el output generado. Por esta causa, la teoría de la señalización cuestiona el elevado gasto que se dedica en todos los países a financiar la educación, lo que de confirmarse tendría serias consecuencias sociales y políticas. Por el contrario, desde el punto de vista de la тсн, la educación es rentable para los individuos y probablemente para la sociedad, ya que el incremento del stock de capital humano se convierte en más productividad y en mayor crecimiento económico (Barceinas \& Raymond, 2003).

Por otro lado, la teoría de la competencia por los puestos de trabajo o de la cola, planteada inicialmente por Thurow (1975), sostiene que la productividad de los trabajadores no depende de sus características personales sino del puesto de trabajo y que los individuos compiten más por estos últimos que por los salarios. En este enfoque, la educación funge como indicador de los costos de formación 
futuros y como herramienta de selección de los empleadores, quienes establecen colas laborales entregando los puestos en función de los costos de formación de los individuos. De esta manera, los trabajadores serán elegidos según sean menores los costos de su formación y por lo tanto los individuos con mayor nivel educativo ocuparán posiciones más ventajosas para acceder a las plazas mejor remuneradas; la educación se percibe aquí como un instrumento para competir.

Por su parte, en la teoría radical, representada por Bowles-Gintis (1976), el papel principal de la educación es el de socializar a los individuos y no tanto el de desarrollar sus capacidades. Desde una perspectiva marxista, en esta teoría se concibe a la educación como un instrumento utilizado por las clases dominantes para mantener su poder segmentando a los trabajadores, y se sostiene que el principal factor de la desigualdad en el ingreso reside en el origen social y no en la dotación de capital humano.

Los planteamientos teóricos mencionados y la evidencia empírica hallada están motivados por "la inequidad salarial", uno de los grandes problemas de las economías, en particular de los países en vía de desarrollo. La evidencia parece indicar que la divergencia educativa ceteris paribus determina la desigualdad salarial. Sin embargo, también muestra que aunque el nivel educativo ha aumentado significativamente eso mismo ha sucedido con la pobreza y la desigualdad salarial (De Ferranti et al., 2003; undp, 2004, 2005, 2006, 2007). Ante esta paradoja, el estudio de los rendimientos educativos demuestra su vigencia.

\section{Los rendimientos de la educación en México}

Existen numerosos estudios empíricos acerca de las derivaciones teóricas sobre capital humano. Y aunque realizados para diferentes países, muestras seleccionadas, variables, periodos, técnicas de estimación y contextos institucionales, su punto de encuentro estriba en que demuestran que el ingreso está determinado por el nivel educativo de los individuos.

Para el caso de México, dichos estudios son amplios y se cińen a los planteamientos teóricos internacionales. Un primer grupo de trabajos que utilizaron datos de las Encuestas Nacionales de Ingresos y Gastos de los Hogares (ENIGH) de los años 1963, 1984, 1989, y las bianuales de 1992 a 2004 y 2005, obtuvieron rendimientos promedio que van del orden del 10 al 16\% (Carnoy, 1967; Psacharopoulos \& Ng. 1992; Psacharopoulos et al., 1996; Psacharopoulos \& Patrińos, 2002; Bracho \& Zamudio, 1994; Lachler, 1998; Rojas, Angulo \& Velázquez, 2000; Barceinas 1999, 2002; Urciaga, 2002; Urciaga \& Vukasinac, 2002; Huesca, 2004; Ríos, 2005; Ordaz, 2007). Por otro lado, solo los trabajos empíricos de Barceinas (2003) y Sariñana (2002) toman en consideración la 
endogeneidad de la escolaridad utilizando las ENIGH de 1994, 1996, el primero, y 1998, el segundo. Las estimaciones de estos autores muestran que los rendimientos de la escolaridad crecen una vez que instrumentan la endogeneidad de esta. Barceinas (2003) aplica primeramente una regresión por partes utilizando la edad como variable instrumental, y luego introduce el producto interno bruto, el presupuesto en educación per cápita en valores constantes y los antecedentes familiares. Mientras que Sariñana (2002) utiliza el número de hermanos y la posición en el empleo del padre. Por último, los estudios de Barceinas $\&$ Raymond (2003) y Mehta \& Villarreal (2008) contrastan la hipótesis de señalización para el caso mexicano con datos de las ENIGH de 1994, 1996, los primeros, y 2002, los segundos. Sus resultados parecen confirmar los postulados de la TCH, pues no encontraron resultados positivos de efecto señalización.

Un segundo conjunto de investigaciones han utilizado los datos de las Encuestas Nacionales de Empleo Urbano (ENEU) del periodo 1987-2004 y así obtuvieron tasas de rendimiento menores, esto es, de entre 6 y $11 \%$ (Zepeda \& Ghiara, 1999; Rodríguez-Oreggia, 2005; López-Acevedo, 2004; Villarreal, 2008). En general tanto este grupo de estudios como el arriba mencionado observan un crecimiento en la tasa de rendimiento promedio de 1984 a 1994. Asimismo coinciden en que los rendimientos asociados a cada nivel educativo son heterogéneos, mostrando que el de los estudios primarios ha disminuido, mientras que el de los superiores ha aumentado en los distintos periodos de tiempo analizados. Las posibles argumentos para explicar estos cambios en los rendimientos se remiten a las reformas institucionales, la apertura comercial y el cambio tecnológico intensivo en conocimiento (Chiquiar, 2004; LópezAcevedo, 2006).

Otros estudios sobre los rendimientos educativos añaden a las estimaciones un componente territorial. Urciaga \& Almendarez (2006) afirman que las regiones más desarrolladas — las cercanas a la frontera, en su estudio- presentan los mayores rendimientos. Barceinas \& Raymond (2005), por su parte, los encuentran en los estados con menos desarrollo, es decir, en los del sur del país. Esto en concordancia con la evidencia empírica internacional, la cual indica que los rendimientos disminuyen en la medida en que aumenta el nivel educativo y el nivel de ingreso per cápita. Otros autores, en cambio, han realizado estimaciones diferenciando entre zonas urbanas y rurales. Por ejemplo, Singh \& Santiago (1997), con base en una muestra de hogares rurales y para 1991, obtienen un rendimiento de $25 \%$ por ańo de escolaridad, en donde las mujeres reportaron 6\% menos que los hombres. Ordaz (2007) halló para el periodo 1994-2005 que la rentabilidad de la educación es más alta en el medio rural que en el urbano en la mayoría de los ańos analizados y en todos los niveles educativos. Además obtuvo que la educación en el medio rural es más 
rentable para las mujeres en los niveles educativos básicos, mientras que para los hombres lo es en los más elevados.

Por último, trabajos más recientes son los de Morales (2011), Harberger \& Peón (2012) y Binelli \& Rubio (2012). Morales (2011) utiliza la Encuesta Nacional sobre los Niveles de Vida de los Hogares (ENNVIH) 2002 para incluir variables de control relevantes en el modelo de Mincer, esto es: un índice de habilidad natural, educación de la madre, infraestructura del hogar durante la etapa escolar, talla y salud. De ese modo concluye que el rendimiento promedio por año de escolaridad se encuentra entre 8.2 y $8.4 \%$. Y en cuanto a los rendimientos marginales por nivel de escolaridad: para primaria $(8 \%)$, para secundaria $(10 \%)$, preparatoria $(2 \%)$, profesional (11\%) y posgrado $(13.85 \%)$. Con ello, la conclusión es que se verifica una relación convexa entre educación y salario.

Por su parte, Harberger \& Peón (2012) utilizan la Encuesta Nacional de Ocupación y Empleo (ENOE) de 2010 y descubrieron un fuerte aumento de las tasas de rendimiento y de los valores presentes netos según los individuos avanzan en su nivel educativo. Estos autores revelaron que las tasas de rentabilidad de los hombres se incrementaron a 2.13, 5.86, 11.26 y $14.27 \%$, de acuerdo a como avanzaron en niveles educativos sucesivos, esto es, pasar de secundaria a bachillerato, de bachillerato a universidad y de universidad a posgrado. Las cifras para las mujeres fueron de 5.49, 7.26, 10.36 y $14.39 \%$, respectivamente.

Por último, Binelli \& Rubio (2012) usaron datos de la Encuesta de Nacional de Trayectorias Educativas y Laborales (ENTELEMS) de 2008 para estimar los rendimientos de la educación media superior (bachillerato) privada. Estos autores no hallaron rendimientos salariales positivos entre los que han estudiado en un bachillerato privado respecto de uno público y que ingresaron al mercado laboral después de graduarse. Aunque sí dan cuenta de un efecto positivo de $54 \%$ en los salarios de aquellos que completaron la educación universitaria.

Los resultados que se han enumerado y que han hallado diversos autores con diferentes metodologías, periodos y bases de datos empleadas para México coinciden en que existe una relación positiva y significativa entre escolaridad, experiencia y salario percibido por hora trabajada; que dicho efecto se eleva sobre el salario con el nivel educativo, y que la relación es mayor para las mujeres. Un trabajo reciente de CEPAL (2016) con datos de 1990 a 2014 muestra que lo anterior puede explicarse en buena parte por varios fenómenos que ocurrieron durante el periodo: 1) un incremento en el nivel de escolaridad promedio (principalmente de las mujeres, las que ahora presentan, en promedio, un mayor nivel educativo), 2) la ampliación de la participación femenina en el mercado laboral (sobre todo en servicios públicos), 3) un producto marginal por hora promedio trabajada de las mujeres 2.7 mayor que el de los hombres, 4) el número total de horas trabajadas por las mujeres es 2.3 veces menor que el de los hombres 
(representa el 30\% del total), y 5) la edad, la experiencia y el salario promedio de las mujeres es menor que el de los hombres.

Esto último, que se refiere a la desigualdad salarial en México, ha sido ampliamente estudiado desde 1980 contrastando distintos enfoques. Y en general se ha encontrado que son tres los factores que inciden sobre esa desigualdad: 1) cambios en la demanda laboral relacionados con la apertura comercial y el cambio tecnológico sesgado e intensivo en conocimiento (Cragg \& Epelbaum 1996; Revenga, 1997; Meza, 1999; Hanson \& Harrison, 1999; Feliciano, 2001; Cañonero \& Werner, 2002; Esquivel \& Rodríguez-López, 2003; Hanson, 2003; Airola \& Juhn, 2005; López-Acevedo, 2004, 2006; Robertson, 2004, 2007); 2) factores de oferta (Chiquiar \& Hanson, 2002; Meza, 2005) y 3) factores institucionales como fuente explicativa (Cortez, 2001; Fairris, 2003; Ghiara \& Zepeda, 2004; Fairris, Popli \& Zepeda, 2008; Bosch \& Manacorda, 2010).

López-Acevedo (2004) y Manacorda, Sánchez-Páramo \& Schady (2010) muestran que en la década de 1980 y en particular en la de 1990 hubo un incremento en la demanda de los trabajadores más cualificados en México debido a la apertura económica y el cambio técnico sesgado a favor de los empleos calificados. Ello pudo haber contribuido a acrecentar los rendimientos educativos. Por otro lado, Urciaga (2004) refiere que la apertura económica acompañada de la menor participación estatal y reducción de la protección comercial, que se dieron a partir de 1994, incrementaron la competencia y provocaron una caída de los salarios reales impulsando los niveles de desigualdad en los ingresos.

Varios trabajos registran el incremento de la desigualdad salarial mexicana en el periodo 1984-1994 explicada por el mayor rendimiento de la calificación tanto con el criterio de ocupación, como con el de escolaridad. Contrario a lo sucedido antes de la firma del TLCAN, la desigualdad salarial en México ha estado disminuyendo después de 1994. La investigación empírica al respecto ha demostrado que los determinantes de tal reducción en la desigualdad corresponden a cambios en la oferta y demanda de trabajo (Lopez-Calva \& Lustig, 2009; Campos, 2010). Por el lado de la oferta se incrementó sustancialmente la tasa de escolaridad en especial en la segunda mitad de los noventa después de la reforma que determinó la obligatoriedad de la educación secundaria. Este incremento en el nivel educativo provocó una reducción importante de la desigualdad salarial después de 1998 mediante la disminución de los rendimientos de la educación. Sin embargo, algunos estudios apuntan que los cambios en la demanda de trabajo calificado han sido insuficientes para compensar el aumento de la oferta de trabajadores con estudios superiores (Lopez-Calva \& Lustig, 2009; Campos, 2010; Esquivel, Lustig \& Scott, 2010; Campos et al., 2012; Tello, Ramos \& Artís, 2012; Lustig, López-Calva \& Ortiz-Juárez, 2013). En este sentido, el mercado de trabajo mexicano no logra absorber la gran cantidad de personas calificadas que ingresan en él. 


\section{Metodología}

Para estudiar la evolución histórica de los rendimientos educativos en México, este artículo se ha fundamentado en el modelo de Mincer (1974). Es el método más utilizado para estimar los rendimientos de la inversión en capital humano y se formuló con base en los supuestos del modelo neoclásico del funcionamiento del mercado de trabajo y de dinero.

Cuando este modelo supone un mercado laboral en competencia implica que las empresas conocen la productividad marginal de cada trabajador y por tanto los salarios se convierten en un reflejo de ella. Asimismo, cuando este modelo considera que el mercado de dinero opera también por competencia, el precio del dinero refleja el coste de oportunidad, por lo que los de la inversión en educación solo corresponden a los de oportunidad, esto es, a los ingresos que no se perciben por estar estudiando.

Respecto de los individuos, el modelo de Mincer considera que son neutrales al riesgo, homogéneos (poseen habilidades innatas y oportunidades similares, y se diferencian por la cantidad de capital humano acumulado), entran de inmediato al mercado laboral al finalizar sus estudios, permanecen en este de manera continua independientemente del nivel de estudios alcanzado, trabajan a tiempo completo y tienen perfecta certidumbre por lo que conocen los ańos de trabajo que les restan en su ciclo de vida. El modelo se representa de la siguiente manera:

Ln $W=\beta_{0}+\beta_{1} S_{1}+\beta_{2} X_{1}+\beta_{3} X_{2}+\alpha_{1} D_{1}+\mu$

donde $L n W$ es la medida de la productividad laboral, expresada mediante el logaritmo natural de los salarios individuales por hora trabajada; $S$ es una medida aproximada de la educación formal, expresada mediante el número de años de estudio finalizados por la población; $X_{1}$ y $X_{2}$ son la experiencia laboral y su cuadrado, respectivamente, calculadas mediante la "experiencia potencial", ya que esta no se puede observar directamente, esto es, la diferencia entre la edad del individuo, el número de ańos de estudio y la edad obligatoria para ingresar a la educación primaria $(6) ; D_{1}$ es una variable correspondiente al sexo del individuo, y toma el valor de 0 si es mujer, y 1 si es hombre; $\mu$ es la perturbación aleatoria; el parámetro $\beta_{0}$ es el intercepto; $\beta_{1}$ mide el incremento porcentual de los salarios (por hora) debidos al incremento de un ańo adicional de educación, por tanto esta relación positiva implicaría que un mayor nivel de capital humano de los trabajadores elevaría su productividad y se traduciría en un mayor salario; $\beta_{2}$ y $\beta_{3}$ teniendo en cuenta la concavidad de los perfiles de edad-ingreso (a medida que se incrementa la experiencia los ingresos individuales también aumentan, pero cada ańo de experiencia tiene 
un efecto sobre los ingresos menor que el anterior) indicará los rendimientos decrecientes de los ingresos en relación con la experiencia, lo que quiere decir que hay una edad en la que se maximizan los ingresos y por tanto se espera que $\beta_{2}$ sea positivo y $\beta_{3}$ negativo.

La literatura sugiere que las estimaciones del rendimiento de la educación por mínimos cuadrados ordinarios (MCO), producto del modelo de Mincer, pueden presentar varios problemas (Griliches, 1977), a saber: 1) la omisión de variables relevantes que pueden producir un error de especificación en la ecuación; 2) el sesgo de selección, ya que solo se analizan los ingresos salariales de los individuos ocupados en la muestra, lo que da lugar a que las estimaciones por MCO sean inconsistentes y sesgadas. ${ }^{3}$

Una de las críticas más frecuentes sobre la estimación de la ecuación minceriana es que la educación se ha considerado habitualmente como una variable exógena, por lo que puede estar afectada por factores que estén fuera del modelo y que a su vez se correlacionen con los residuos y den lugar a estimaciones inconsistentes incurriendo en el denominado sesgo de endogeneidad o de habilidad.

Para corregir este problema, diversos estudios han utilizado variables instrumentales (VI) como alternativa. Esto consiste en echar mano de variables que influyan sobre la escolaridad, pero que no se relacionen con los determinantes omisos de los salarios o con el error de medición en el nivel educativo. Esas variables se clasifican en dos grupos: las referentes al entorno familiar o background (muestras de gemelos, educación y características socioeconómicas de los familiares), y las relativas a las variaciones naturales (proximidad a la escuela, costos de colegiatura, fecha de nacimiento, factores institucionales, afectación de reformas educativas, etc.). ${ }^{4}$ Aquí, como para tratar el sesgo de endogeneidad no se cuenta con instrumentos relacionados con el background familiar o con la habilidad de los individuos, se han empleado instrumentos de tipo institucional. Para ello se tomó como referencia a Harmon \& Walker (1995) y Acemoglu \& Angrist (2000), quienes consideran las leyes de escolaridad obligatoria como un determinante exógeno del nivel educativo alcanzado.

En esta investigación, el interés se enfoca en el efecto que pueda tener sobre las posibilidades de estudio la implantación general de la enseñanza secunda-

3 Heckman (1997) propone corregir este problema con un procedimiento en dos etapas: en la primera un modelo probabilístico determina la posibilidad de que un individuo participe en el mercado de trabajo y, en la segunda, introduce la ratio inversa de Mills. Con la estimación de la primera, y a partir de los residuos, se incorpora como variable independiente adicional en la función de ingresos.

4 Ginther (2000) recurre a la utilización de la estructura familiar, la proximidad a la escuela y la calidad de esta última como instrumentos. Angrist \& Krueger(1991), Angrist \& Imbens (1995) y Card (1993) recurren a la fecha de nacimiento y a la cercanía de la escuela como instrumentos. 
ria obligatoria gratuita. En México, al declararse como obligatorio este nivel educativo la escolaridad básica se amplió de seis a nueve años, con el apoyo de las reformas al artículo tercero de la Constitución y de la promulgación de la nueva Ley General de Educación (LGE) aprobadas en 1993 por el Congreso de la Unión. En resumen, se ha creado en esta investigación una variable que controla si los individuos se encuentran o no afectados por dicha reforma. Aunque debe agregarse que otra de las críticas al modelo minceriano es la medición incorrecta de la cantidad de educación.

Como se mencionó arriba, la ecuación (1) supone que el capital humano es homogéneo y que existe una tasa única de rendimiento de la educación, sin embargo, teoría y evidencia empírica muestran lo contrario. Por lo tanto, es posible que la aproximación se estuviera dando al rendimiento educativo de los subgrupos de la población caracterizados por un mayor rendimiento y no a los de un rendimiento educativo promedio, lo que a la postre provocaría una infraestimación en el verdadero rendimiento. Por lo anterior se decidió representar la escolaridad en forma más desagregada con el fin de representar más adecuadamente los retornos de la inversión en educación (Sapelli, 2009). Así, en tercer término, se procedió a sustituir la variable años de escolaridad $S$ de la función minceriana por cinco variables ficticias correspondientes a los distintos niveles educativos obtenidos, tomando el valor de 1 para el individuo que pertenece a esa categoría y de 0 para los demás casos.

$$
\text { Ln } W=\beta_{0}+\beta_{1} X_{1}+\beta_{2} X_{2}+\alpha_{1} D n_{1}+\alpha_{2} D n_{2}+\alpha_{3} D n_{3}+\alpha_{4} D n_{4}+\alpha_{5} D n_{5}+\mu
$$

donde la primera variable generada es $D n_{0}$ cuando la persona no ha cursado ningún grado escolar y constituye nuestra categoría de referencia; $D n_{1}$, Primaria si la persona ha cursado de 1 a 6 ańos de escolaridad; $D n_{2}$, Secundaria, si la persona ha cursado de 7 a 9 ańos; $D n_{3}$, Bachillerato, si ha estudiado de 10 a 12 ańos; $D n_{4}$, Universidad, si ha cursado de 13 a 16 ańos, y $D n_{5}$, Posgrado, cuando el individuo ha estudiado 17 años o más.

Los resultados de los coeficientes estimados de la ecuación (2) se han utilizado para calcular los rendimientos absolutos y marginales. Los primeros se obtienen con la fórmula $r_{i}=\beta_{i} / n$, donde $i$ representa cada uno de los niveles educativos y $n$ el número de años de estudio requerido para terminarlo. ${ }^{5}$ Es decir, los rendimientos absolutos provienen de dividir los coeficientes obtenidos en cada nivel educativo entre el número de años requeridos para terminarlos, y por ello se refieren al comparativo entre los individuos sin estudios en relación con

$5 \quad n_{i}$ : Primaria (6), Secundaria (9), Bachillerato (12), Universidad (16) y Posgrado (18). 
los demás niveles educativos. Por su parte, los rendimientos marginales se consiguieron mediante la expresión $r_{i}=\left(D_{i}-D_{i-1}\right) / n_{i}$, tomando la propuesta de Psacharopoulos (1994). En ella se compara la diferencia entre los coeficientes obtenidos entre dos niveles educativos y se divide por el número de años de estudio necesarios para pasar de un nivel al siguiente ${ }^{6} \mathrm{y}$, en consecuencia, se refiere al diferencial respecto del nivel educativo anterior.

\section{Datos}

En esta investigación se han utilizado datos de la Encuesta Nacional de Empleo (ENE) ${ }^{7}$ del periodo 1995-2004, y de la Encuesta Nacional de Ocupación y Empleo (ENOE) de 2005 al 2012, debido a que desde 2005 esta última reemplazó a la primera.

La información de la ENE hasta diciembre de 2004, tal y como se difundió en su momento, no es comparable con la de la ENOE, por ello el INEGI homologó las series de aquella siguiendo los criterios de la segunda, y el resultado fue el sistema para la consulta de indicadores estratégicos (InfoLaboral). Este contiene información armonizada de 1995 a la fecha con actualización trimestral para los diversos ámbitos de cobertura geográfica (nacional, por entidad federativa y cuatro tamańos de localidad), dependiendo del año seleccionado. En este artículo se ha usado la información ya armonizada metodológicamente, lo cual permite los comparativos de variables, ya sea entre dimensiones geográficas, o temporales, es decir, entre trimestres. De igual modo, con el objetivo de homogeneizar la muestra seleccionada en ambas encuestas se han seleccionado solo las 32 áreas metropolitanas de las que hay registro en todo el periodo analizado.

De toda la información de dichas encuestas fue seleccionado solo el tercer trimestre de cada año, respectivamente, ya que es el de mayor uso en este tipo de trabajos por ser el de más estabilidad económica. La muestra se restringió a la población ocupada entre 14 y 65 años de edad con ingresos a sueldo fijo y que hubieran laborado entre veinte y sesenta horas en la semana de referencia.

Después de depurar la información, la base de datos quedó conformada con más de un 1111000 observaciones, de las que el 62\% fueron hombres y el 38\%

$6 \quad n_{i}$ : Primaria (6), Secundaria (3), Bachillerato (3), Universidad (4) y Posgrado (3).

7 La ENE nace en 1988 y se publicaban sus resultados anualmente pero, ante la necesidad de dar mayor peso a las estadísticas sobre ocupación y de ampliar la periodicidad de las cifras con representatividad nacional y estatal, se determinó levantarla de manera continua y publicar sus resultados trimestralmente. Así, desde mayo de 2003 se dispone de microdatos sobre la situación laboral del país con representatividad nacional para cuatro tamaños de localidad de las 32 entidades federativas y una ciudad en cada una de ellas, con una serie estadística que abarca información desde el segundo trimestre de 2000. 
mujeres. Los hombres promediaron 36 años de edad, 9.9 años de escolaridad, 19.7 ańos de experiencia, 45.5 horas de trabajo a la semana y salario de $\$ 25.6$ M. N. por hora (tabla 1). Las mujeres promediaron 35 años de edad, 10.2 años de escolaridad, 18.8 años de experiencia, 41.2 horas de trabajo a la semana y salario de $\$ 22.7 \mathrm{M}$. N. por hora (tabla 1 ). Se realizaron pruebas $t$ de diferencias de medias en las variables clave de edad, escolaridad, experiencia, horas trabajadas y salario por hora entre hombres y mujeres en el total de la base de datos. En las cinco variables citadas se obtuvieron diferencias estadísticamente significativas entre hombres y mujeres, razón por la que se decidió realizar todas las estimaciones con el total de las observaciones y posteriormente por separado para hombres y mujeres a fin de asegurar significatividad estadística.

Tabla 1. Promedios de las variables clave de la base de datos utilizada

\begin{tabular}{lcccccc}
\hline & Observaciones & Edad & Escolaridad & Experiencia & $\begin{array}{c}\text { Horas } \\
\text { trabajadas }\end{array}$ & $\begin{array}{c}\text { Salario } \\
\text { por hora }\end{array}$ \\
\hline Total & 1111048 & 35.340 & 10.014 & 19.325 & 43.892 & 24.542 \\
Mujeres & 420905 & 34.985 & 10.198 & 18.786 & 41.234 & 22.742 \\
Hombres & 690143 & 35.556 & 9.902 & 19.654 & 45.513 & 25.640 \\
Diferencia & & -.57113 & .29641 & -.86755 & -4.2787 & -2.8980 \\
de medias & & $(-24.71)$ & $(32.30)$ & $(-34.131)$ & $(-2.4 \mathrm{e}+02)$ & $(-48.857)$ \\
\hline
\end{tabular}

Nota: Valor del estadístico t entre paréntesis.

Fuente: Elaboración propia con base en la ENE (1995-2004) y ENOE (2005-2012).

\section{Resultados}

\section{Rendimientos promedio de la educación}

A fin de superar los problemas de heteroscedasticidad — muy común en estimaciones con datos de corte transversal-, se llevaron a cabo las estimaciones empleando el procedimiento de White, el cual calcula matrices de covarianzas robustas a cualquier tipo de heteroscedasticidad para arribar a estimaciones consistentes. Por otro lado, como señalan Barceinas et al. (2003), es usual que en modelos con datos microeconómicos de sección cruzada, el $R^{2}$ de la ecuación estimada por mínimos cuadrados ordinarios suele encontrarse en torno al 30\% como sucede en el caso que aquí se presenta. Por ello, entre el 25 y el $40 \%$ de la variación de los salarios se explica por las variables del modelo, quedando sin explicar fuera entre 60 y $75 \%$. Para el periodo de tiempo analizado apareció una reducción en la magnitud de este indicador, lo cual significa que la capacidad del modelo para explicar la variación en los salarios de los individuos también se ha reducido. De manera contraria, el valor del término constante se incrementó para el periodo en análisis (ingreso predeterminado para los trabajadores sin estudios). 
Los resultados de las estimaciones mincerianas para los años en estudio indicaron que todos los coeficientes de las variables son estadísticamente significativos al $99 \%$ de confianza y con el signo y la magnitud esperados, lo que es consistente con las estimaciones habituales del modelo básico de capital humano. De este modo, la variable aproximada de experiencia mostró una relación positiva en el nivel de salarios, mientras que el coeficiente negativo de la experiencia al cuadrado señaló una relación decreciente y, por esto, un punto máximo en el que los individuos maximizan dichos ingresos. En cuanto a la principal variable de interés, esto es, la relativa al nivel educativo, hubo una relación positiva entre capital humano individual y salarios. Así, los resultados de los rendimientos promedio de la educación en el periodo 1995-2012 indican que por cada año adicional de estudios el ingreso promedio de los individuos se incrementa en torno al $9 \%$.

$\mathrm{Al}$ tomar en consideración el género en el periodo analizado, se obtuvo que en promedio los hombres presentan mayor edad, experiencia, horas trabajadas y un salario por hora $13 \%$ superior al de las mujeres. No obstante, los hombres también poseen un menor número de años de escolaridad promedio y un rendimiento educativo 19\% menor que el de las mujeres, lo cual indicaría que los ingresos de las mujeres se incrementarían por cada año adicional de estudios en una proporción superior a la de los hombres, mostrando de esa manera un mayor diferencial de ingresos en comparación con los hombres. Los resultados de esta investigación coinciden con los de otros trabajos previos para México.

Otro hallazgo acerca de la evolución en el tiempo de los rendimientos promedio es que se distinguen dos periodos (gráfica 4). En el primero se nota una débil tendencia decreciente que llega hasta 2004, pero a partir de 2005 se da una pequeña recuperación en los coeficientes de los rendimientos. Esta reducción contrasta con el comportamiento de décadas anteriores.

Gráfica 4. Rendimientos promedio de la educación en México, 1995-2012

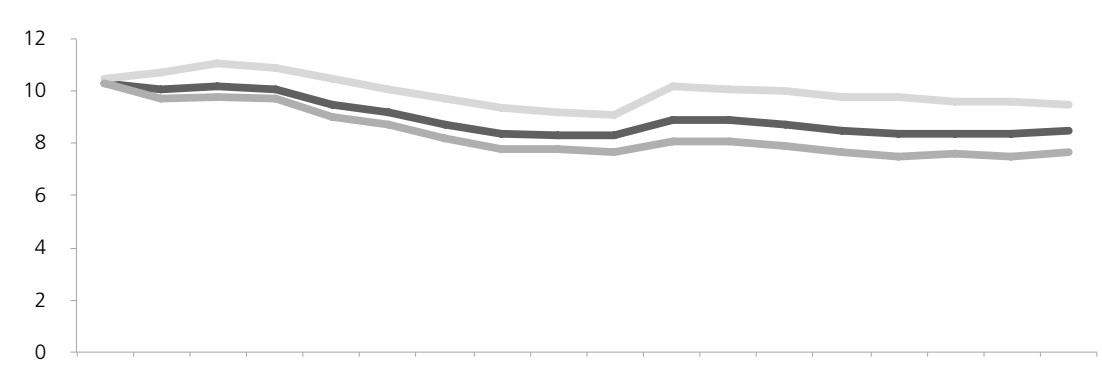

199519961997199819992000200120022003200420052006200720082009201020112012

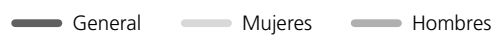

Fuente: Elaboración propia con base en la tabla 2 del anexo. Ecuación estimada: $L n W=\beta 0+\beta 1 S+\beta 2 X I+\beta 3 X 2+$ $+\alpha 1 D I+\mu$ (l) 
Las estimaciones del rendimiento educativo obtenidas por MCO a partir de la ecuación minceriana de ingresos pueden presentar sesgos debidos a la endogeneidad de la educación. Las dos principales fuentes de dicho sesgo son la habilidad, que haría que los MCO sobreestimen el rendimiento y el de error de medida en la educación, que haría que los MCO infraestimen el verdadero rendimiento. Para evaluar el posible impacto de estos sesgos sobre los resultados, se llevaron a cabo estimaciones del rendimiento de la educación con variables instrumentales de tipo institucional. Para ello se tomaron como base a Harmon \& Walker (1995) y Acemoglu \& Angrist (2000), autores que consideran las leyes de escolaridad obligatoria como un determinante exógeno del nivel educativo alcanzado que afecta la elección de educación de los individuos pero no sus ingresos.

Siguiendo la misma línea ya planteada, se utilizó como instrumento una variable que reflejara si el individuo se ha visto afectado o no por el cambio legislativo en el nivel de escolaridad obligatorio. En primer lugar y para determinar la potencial endogeneidad de la escolaridad se aplicó el test de Hausman.

Los resultados de las estimaciones promedio y las de los hombres condujeron a rechazar en todos los casos, excepto para 2010 y 2011, a un nivel de confianza del $95 \%$, la hipótesis nula de que el nivel de escolaridad está determinado exógenamente. Lo anterior justifica el método de mínimos cuadrados en dos etapas utilizando variables instrumentales mostrándolo como apropiado para calcular los rendimientos de la educación. Los resultados de la prueba estadística señalaron que, para las mujeres, la hipótesis nula de que el nivel de escolaridad sea exógeno se rechaza en aproximadamente la mitad de los eventos para los años del estudio.

Seguidamente y previo a la interpretación de los resultados de las estimaciones con VI cabe considerar la calidad y validez de los instrumentos empleados. En cuanto al primer aspecto, se usó el contraste de Bound, Jaeger \& Baker (1995). Con esa intención se presentan en la tabla 3 del anexo el valor " $F$ de los instrumentos excluidos" y el " $R^{2}$ parcial del instrumento", obtenidos a partir de la primera etapa de la estimación, justo el momento en que se estimó una regresión por MCO donde la escolaridad es la variable dependiente, exógenas las demás variables explicativas (experiencia, experiencia al cuadrado y sexo, según sea el caso) y donde la variable instrumental (la reforma educativa de 1993) actúa como variable independiente. En la segunda etapa se recurrió a los valores de los residuos obtenidos incluyéndolos como variables explicativas de la ecuación original de ingresos. Los resultados del valor del estadístico $F$ indicaron en todas las estimaciones la significatividad conjunta de los instrumentos 
excluidos en la primera etapa, sugiriendo así como adecuado el uso de la reforma educativa de 1993 como instrumento. Sin embargo, respecto de la " $R^{2}$ parcial del instrumento" se consiguieron los valores más bajos en las estimaciones del periodo 1995-2000. Esto tal vez se deba a que durante estos primeros ańos la proporción de afectados por la reforma educativa es reducido, y a que su efecto en el mercado laboral y en los ingresos de los individuos tarda en manifestarse. Sobre la validez de los instrumentos, el test de Sargan de restricciones sobreidentificadas probaría la hipótesis nula de que dichos instrumentos no están correlacionados con el término de error y que el uso de estas variables es adecuado. Desafortunadamente no ha sido posible realizarlo debido a que la ecuación está exactamente identificada.

Gráfica 5. Rendimientos de la educación por variables instrumentales

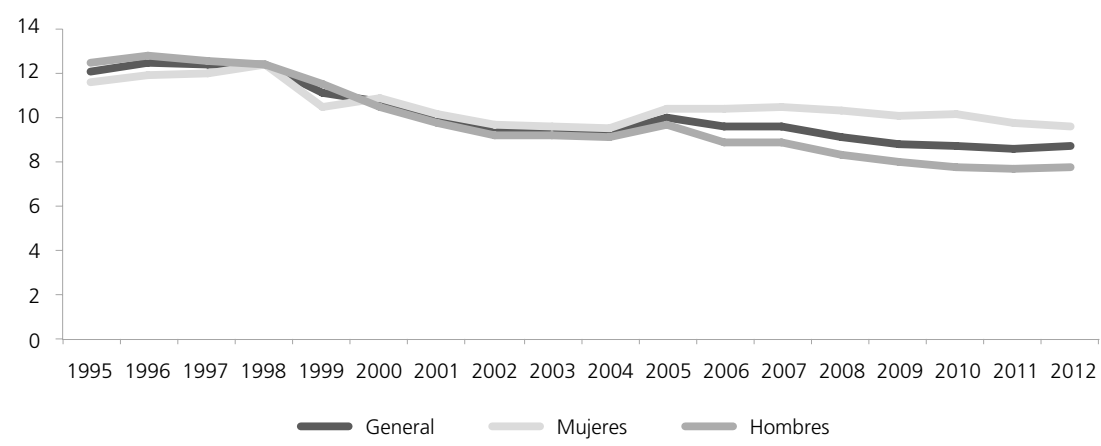

Fuente: Elaboración propia con base en la tabla 3 del anexo.

Los resultados se presentan en la gráfica 5 . Una vez que se controla el problema de la endogeneidad de la educación por medio de variables instrumentales, ellos muestran que los rendimientos estimados se incrementan alrededor de $12 \%$ cuando se comparan con los que arrojan los mco. Podría decirse entonces que los MCo estarían sesgando a la baja el valor del rendimiento, y que por esto predomina el error en la medición de la escolaridad. Ello se corresponde con otros trabajos sobre el tema (Barceinas, 2003; Sariñana 2002). A fin de hallar una explicación sobre esto, Card $(1999,2000)$ sugiere la presencia de heterogeneidad en los rendimientos. Esto es, que los rendimientos provenientes de las estimaciones por VI son una media ponderada de los individuos cuyas decisiones de educación se ven afectadas por los instrumentos y no el rendimiento marginal medio de la población como lo muestran las estimaciones por MCo. Se 
deduce de la estimación que por la reforma educativa los individuos aumentaron su nivel de escolaridad y consiguieron un mayor rendimiento que el resto. En consecuencia, posiblemente se estaría modificando la decisión de educación de las personas con bajo nivel educativo y probablemente por esto tendrían mayores rendimientos que la media. Es razonable afirmar que los rendimientos por VI superan a los de MCO, ya que se estaría capturando la rentabilidad de la inversión en educación de este subgrupo de individuos. Así, la interpretación de Card en cuanto a que las estimaciones por VI son superiores a las de MCO sería coherente con la propuesta de Angrist \& Imbens (1995) conocida como "efecto tratamiento promedio local" (LATE).

\section{Rendimientos por niveles educativos}

Con el propósito de controlar la heterogeneidad en los rendimientos de la escolaridad se llevaron a cabo estimaciones desagregadas por niveles educativos para la muestra en general y por género. Pudo observarse entonces que los rendimientos educativos contienen diferencias importantes en especial en los niveles más altos, esto es en universidad y posgrado. Esto quiere decir que no hay una tasa única u homogénea de rendimiento de la inversión en educación, sino que varía entre niveles educativos.

Los resultados en cuanto a los niveles educativos indican que el rendimiento en todos ellos reporta coeficiente positivo y creciente al aumentar la escolaridad, lo cual es normal para este tipo de ecuaciones. Conforme los individuos logran más educación incrementan su productividad y perciben mejores salarios; cuanto más alto es el nivel educativo de los individuos, mayor es la compensación salarial que consiguen y por tanto los estudios de posgrado son los que muestran las más altas tasas de rendimientos.

La gráfica 6 sintetiza los resultados de los coeficientes de la estimación por niveles educativos tomando como referencia la categoría de las personas sin estudios e indicando el diferencial salarial entre las personas sin estudios y el resto de los niveles. Es decir, que una persona con estudios de primaria recibe aproximadamente $24 \%$ más que una persona sin estudios. Los individuos con secundaria percibieron 53\% más que aquellos sin estudios. Quienes cuentan con bachillerato ganaron $86 \%$ más que alguien sin estudios. Los universitarios reportaron un diferencial salarial de $143 \%$. En tanto que aquellos con estudios de posgrado muestran un diferencial salarial de $193 \%$ más respecto de la persona sin estudios. Una observación que deriva de estos resultados es que en México estudiar es rentable, puesto que todos los niveles educativos ofrecen rentabilidades positivas en todos los años analizados. 
Gráfica 6. Coeficientes estimados de la regresión por niveles educativos

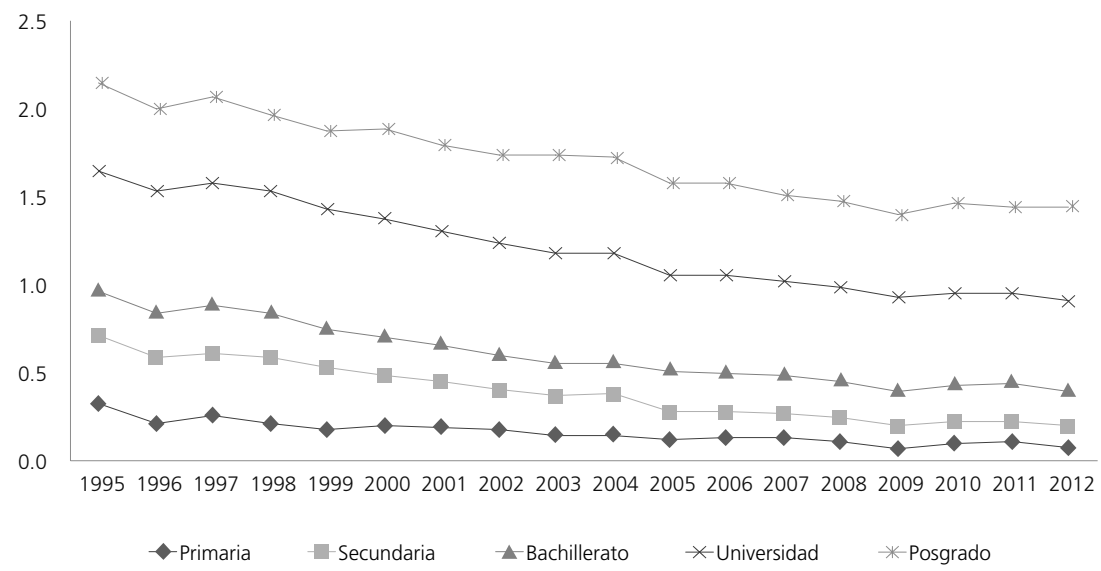

Fuente: Elaboración propia con base en la tabla 4 del anexo.

Los rendimientos por niveles educativos en términos absolutos y marginales decrecieron entre 1995 y 2012 (tabla 5 del anexo). Aunque las tasas de rendimiento absolutas y marginales de la educación son mayores en los niveles de educación superior. Esto quiere decir que el beneficio de invertir en un año adicional de estudio es menor para los individuos menos educados. Además la brecha en términos de rentabilidad educativa absoluta y marginal se ha reducido para los niveles educativos inferiores, mientras que para los más altos, universidad y posgrado, se ha mantenido relativamente estable.

Al estimar por género, las mujeres consiguen una mayor rentabilidad educativa que los hombres en la mayoría de los años estudiados y en todos los niveles educativos. De acuerdo con esto, los resultados principales se mantienen y las estimaciones son robustas desde el punto de vista estadístico: las mujeres obtienen en promedio mayor rentabilidad de la educación en la mayoría de los años analizados que los hombres y los rendimientos decrecen en el periodo de estudio. Este comportamiento se encuentra en línea con la evidencia internacional. Esto es, que las tasas de rendimiento de la educación disminuyen conforme crece el promedio de educación ${ }^{8}$ y el ingreso per cápita, ${ }^{9}$ ambas reflejadas para

8 El número de años de escolaridad promedio de la muestra fue de 8.6 en 1995 y de 10.6 en 2012. Lo cual significa un incremento del $22 \%$ en el periodo, y una tasa de crecimiento promedio anual de $1.25 \%$.

9 El ingreso individual muestra un crecimiento del 57\% en el periodo analizado, pasando de 1.74 en 1995 a 3.27 en el 2012, con un crecimiento promedio anual de 3.19 . 
México. Sin embargo, la evidencia de estos dieciocho años de estudio indica un descenso más grande en los rendimientos de los niveles educativos inferiores (primaria, secundaria y bachillerato). Conforme avanza el tiempo, la tendencia del mercado de trabajo sigue premiando salarialmente más a los que tienen niveles superiores de estudio.

Pero, contrario a la evidencia empírica del periodo previo a 1995 cuando había una tendencia creciente de los rendimientos educativos, esta se ha reducido por varios factores, entre los que se cuenta que la economía mexicana experimentó en los últimos veinte años cambios notables que han incidido en el mercado laboral. Este ha sido el papel de las reformas económicas, institucionales, demográficas, la apertura externa y el cambio tecnológico intensivo en conocimiento. Diversos trabajos afirman que la caída de los rendimientos se explica por el incremento en la oferta de trabajadores calificados y el estancamiento o reducción de la demanda relativa de trabajo cualificado. Asimismo, el crecimiento de esta oferta relativa se debe al establecimiento de la educación básica como obligatoria, lo que ha reducido la cantidad de trabajadores menos calificados. Es así que la distribución del capital humano se ha uniformado reduciendo la brecha en los rendimientos de la educación por niveles (Esquivel, Lustig \& Scott, 2012; Lustig et al., 2013). Sin embargo, los cambios en la demanda de trabajo calificado no han sido suficientes para compensar la creciente oferta de trabajadores con estudios superiores.

\section{Gráfica 7. Rendimientos absolutos por niveles educativos en México}

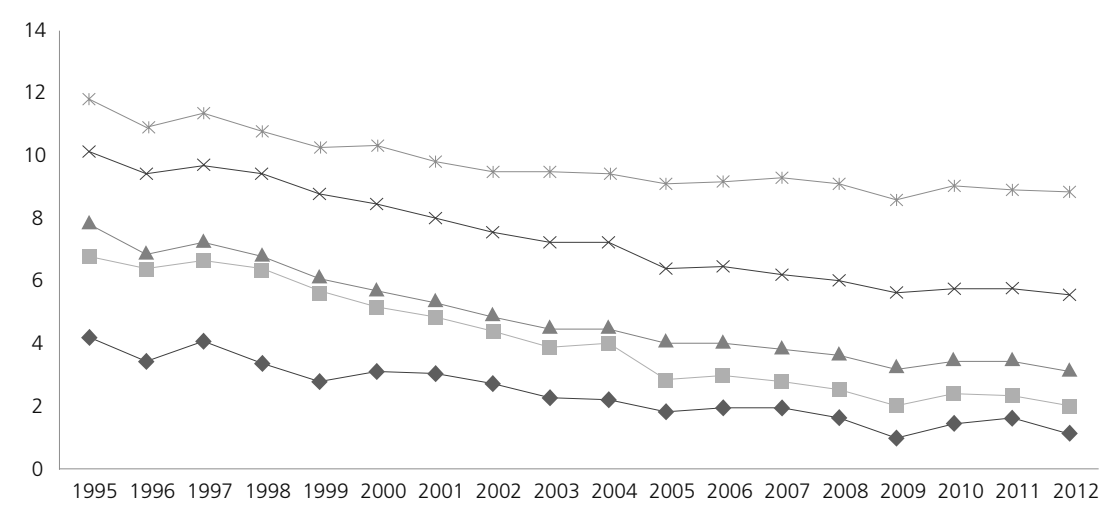

--Primaria - - Secundaria $\quad *$ - Bniversidaderato $\quad *$ Posgrado

Nota: Los rendimientos absolutos se obtienen respecto de la categoría sin estudios.

Fuente: Elaboración propia con base en la tabla 5 del anexo. 


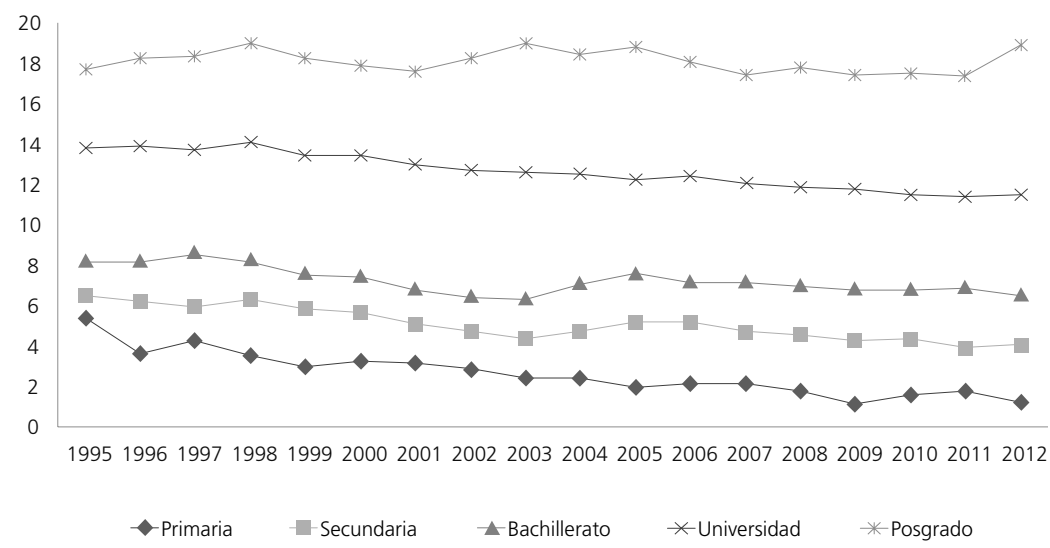

Nota: Los rendimientos marginales se obtienen respecto del nivel educativo previo.

Fuente: Elaboración propia con base en la tabla 5 del anexo.

\section{Conclusiones}

En este trabajo se han calculado las tasas de rendimiento de la educación en México estimando la ecuación básica minceriana para hombres y mujeres tratando de controlar los sesgos que eventualmente aparecen en la estimación que se realiza con Mco. Es por esto que se ha considerado la posible endogeneidad de la educación estimando por variables instrumentales y teniendo en cuenta los diversos niveles educativos.

De esta forma, para el periodo de estudio los resultados fueron rendimientos promedio de la educación del $9 \%$ con la ecuación minceriana estimando por Mco, los cuales, además de reducirse, observaron diferencias de hasta casi tres puntos en sus valores cuando se controló por género.

Posteriormente, cuando se consideró la endogeneidad de la escolaridad utilizando una variable que indica si el individuo se ha visto afectado o no por la reforma educativa de 1993 que impuso como obligatoria la educación secundaria, el hallazgo fue que los rendimientos se incrementan en $10.1 \%$, es decir, que aumentan $12 \%$ respecto de los rendimientos por MCO, lo que coincide con otros trabajos previos sobre el tema.

La estimación del rendimiento de la educación al emplear la técnica de variables instrumentales puede interpretarse como el rendimiento marginal promedio del grupo de individuos cuya decisión de educación se ve afectada por el 
fenómeno que refleja el instrumento. Esta estimación podría no representar el rendimiento promedio de la educación sino el de un grupo particular situado lejos de la media, pero fuertemente correlacionado con el instrumento utilizado. La reforma educativa de 1993 provocó un significativo incremento en el nivel de escolaridad de los que vivieron su proceso educativo en este nuevo marco, por lo cual el parámetro obtenido puede ser de gran utilidad en la evaluación de políticas económicas. Se debe esperar que la más reciente reforma que determinó como obligatoria la educación media superior, que entró en vigor en el ciclo 2012-2013 y que facilita el acceso a mayor educación, genere un efecto similar en la población.

Asimismo, en esta investigación se ha descompuesto la variable escolaridad con variables dummy que corresponden a los distintos niveles educativos. Así se ha encontrado que los rendimientos de la educación crecen según se incrementen los años de escolaridad. Por esto es posible afirmar que los estudios de posgrado, para hombres y mujeres, son las inversiones educativas más rentables.

Es importante enfatizar que, hechas las estimaciones, se observa que independientemente del año de estudio y del nivel educativo, los rendimientos promedio de las mujeres son más altos que los de los hombres, aunque parten de un nivel salarial más bajo. Los resultados señalan que la inversión en capital humano, en su modalidad de más educación formal, es determinante para lograr mayores ingresos salariales en México. Asimismo, al considerar la tasa de rentabilidad marginal se demuestra que transitar de un nivel educativo a otro aumenta también la remuneración de los trabajadores. Esto a final de cuentas sería un incentivo para que las personas busquen elevar su nivel de escolaridad para mejorar sus posibilidades de acceso al empleo formal.

La evolución de los rendimientos educativos durante el periodo estudiado muestra una asociación con los cambios en el tiempo de la oferta y la demanda de trabajo calificado. La caída que se observa en los rendimientos tal vez tenga su causa en el incremento de la oferta de trabajadores calificados y el estancamiento o reducción de la demanda relativa del trabajo cualificado. Cabe agregar que el crecimiento de dicha oferta relativa tiene que ver con la ampliación de la cobertura de la educación básica obligatoria derivada de la implementación de la reforma educativa; esto ha reducido la cantidad de trabajadores con menos cualificación. Esta investigación también ha detectado una disminución en la demanda de trabajadores con educación universitaria. Esto quiere decir que la distribución del capital humano se ha hecho más uniforme reduciendo la brecha en los rendimientos de la educación por niveles, y que los rendimientos de la educación universitaria son más bajos que en 1995. A pesar de que este comportamiento de los rendimientos ha traído ventajas como la disminución 
de la desigualdad salarial, el hecho puede verse como algo positivo para la sociedad. Es un descenso, desde luego, con inconvenientes como, por ejemplo, que los nuevos graduados de la universidad no han hallado puestos de trabajo de acuerdo a la formación que han recibido.

\section{Anexo}

Tabla 2. Rendimientos de la educación, 1995-2012. MCO: robustos a heteroscedasticidad

\begin{tabular}{|c|c|c|c|c|c|c|c|c|c|}
\hline & 1995 & 1996 & 1997 & 1998 & 1999 & 2000 & 2001 & 2002 & 2003 \\
\hline \multirow[t]{2}{*}{ Escolaridad } & $0.103^{\star \star \star}$ & $0.101^{\star \star *}$ & $0.102^{* * *}$ & $0.101^{* * *}$ & $0.095^{\star * *}$ & $0.092^{* * *}$ & $0.087^{\star * *}$ & $0.084^{* \star *}$ & $0.083^{\star \star \star}$ \\
\hline & (106.3) & (186.4) & (116.1) & (198.7) & (104.5) & (189.6) & $(184.0)$ & (178.75) & (161.8) \\
\hline \multirow[t]{2}{*}{ Experiencia } & $0.041^{\star \star *}$ & $0.040^{\star \star *}$ & $0.039^{* * *}$ & $0.040^{\star * *}$ & $0.036^{* * *}$ & $0.034^{\star * *}$ & $0.033^{\star \star *}$ & $0.032^{\star \star \star}$ & $0.032^{\star * *}$ \\
\hline & (37.98) & (64.52) & $(38.41)$ & $(66.70)$ & (33.78) & $(62.48)$ & (60.93) & (59.32) & (56.01) \\
\hline \multirow[t]{2}{*}{ Experiencia 2} & $-0.00^{\star \star \star}$ & $-0.01^{\star * \star}$ & $-0.00^{\star \star \star}$ & $-0.00^{\star \star \star}$ & $-0.00^{\star * *}$ & $-0.00^{\star * \star}$ & $-0.00^{\star * *}$ & $-0.00^{\star * *}$ & $-0.00^{\star \star *}$ \\
\hline & $(-23.46)$ & $(-37.52)$ & $(-21.72)$ & $(-39.22)$ & $(-19.95)$ & $(-36.75)$ & $(-36.42)$ & $(-35.34)$ & $(-33.77)$ \\
\hline \multirow[t]{2}{*}{ Sexo } & $0.074^{\star * *}$ & $0.097^{\star * *}$ & $0.110^{* * *}$ & $0.116^{\star * *}$ & $0.113^{\star \star *}$ & $0.156^{\star * *}$ & $0.171^{\star * *}$ & $0.161^{* \star \star}$ & $0.163^{\star \star \star}$ \\
\hline & $(9.14)$ & (20.26) & $(13.86)$ & $(25.40)$ & (13.79) & (37.34) & $(41.98)$ & (39.20) & (37.10) \\
\hline \multirow[t]{2}{*}{ Constante } & $0.310^{\star * *}$ & $0.448^{\star \star \star}$ & $0.639^{* * *}$ & $0.796^{\star * *}$ & $1.035^{\star * *}$ & $1.288^{* * *}$ & $1.450^{\star \star \star}$ & $1.567^{\text {*** }}$ & $1.618^{\star \star *}$ \\
\hline & (21.01) & (51.43) & $(45.00)$ & (96.09) & $(70.06)$ & (167.39) & (188.82) & (203.05) & (191.54) \\
\hline Observaciones & 27566 & 70436 & 24879 & 77310 & 22649 & 91418 & 89459 & 86447 & 76925 \\
\hline$R^{2}$ Ajustado & 0.343 & 0.375 & 0.393 & 0.380 & 0.365 & 0.337 & 0.333 & 0.322 & 0.312 \\
\hline $\mathrm{F}$ & 3109 & 9421 & 3664 & 10887 & 3007 & 10094 & 9606 & 9048 & 7456 \\
\hline
\end{tabular}

Tabla 2. Continuación

\begin{tabular}{|c|c|c|c|c|c|c|c|c|c|}
\hline & 2004 & 2005 & 2006 & 2007 & 2008 & 2009 & 2010 & 2011 & 2012 \\
\hline \multirow[t]{2}{*}{ Escolaridad } & $0.083^{\star \star \star}$ & $0.089^{* \star \star}$ & $0.089^{\star * *}$ & $0.087^{\star * *}$ & $0.085^{\text {***}}$ & $0.084^{\star \star \star}$ & $0.084^{\star \star \star}$ & $0.084^{\star \star \star}$ & $0.085^{\star \star \star}$ \\
\hline & $(136.6)$ & $(144.1)$ & $(143.1)$ & (140.7) & $(134.0)$ & (125.2) & (126.5) & (121.5) & (123.5) \\
\hline \multirow[t]{2}{*}{ Experiencia } & $0.032^{\star \star \star}$ & $0.032^{\star \star \star}$ & $0.030^{* * *}$ & $0.028^{\star * *}$ & $0.028^{* \star *}$ & $0.027^{\text {** }}$ & $0.026^{\star \star \star}$ & $0.026^{\star \star \star}$ & $0.025^{\star \star \star}$ \\
\hline & $(47.51)$ & $(54.42)$ & (52.22) & (49.14) & $(47.72)$ & $(44.19)$ & $(41.83)$ & $(42.81)$ & $(40.52)$ \\
\hline \multirow[t]{2}{*}{ Experiencia 2} & $-0.00^{\star \star \star}$ & $-0.00^{\star \star \star}$ & $-0.00^{\star \star \star}$ & $-0.00^{\star \star \star}$ & $-0.00^{\star * *}$ & $-0.00^{\star \star \star}$ & $-0.00^{\star * *}$ & $-0.00^{* * *}$ & $-0.00^{\star \star \star}$ \\
\hline & $(-29.09)$ & $(-31.05)$ & $(-28.53)$ & $(-26.43)$ & $(-26.56)$ & $(-24.60)$ & $(-22.58)$ & $(-24.12)$ & $(-21.38)$ \\
\hline \multirow[t]{2}{*}{ Sexo } & $0.169^{\star \star \star}$ & $0.189^{* \star *}$ & $0.181^{* * *}$ & $0.177^{\text {***}}$ & $0.186^{* * *}$ & $0.171^{\text {*** }}$ & 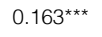 & $0.151^{\text {***}}$ & $0.158^{\star \star \star}$ \\
\hline & (32.81) & $(41.00)$ & (39.94) & $(39.25)$ & $(40.11)$ & $(35.66)$ & $(34.07)$ & (31.19) & (32.79) \\
\hline \multirow[t]{2}{*}{ Constante } & $1.659^{\star \star \star *}$ & $1.630^{\star \star \star}$ & $1.713^{\star \star *}$ & $1.801^{\star \star *}$ & $1.846^{\star \star \star}$ & $1.867^{* \star *}$ & $1.909^{\star \star \star}$ & $1.934^{\star \star \star}$ & 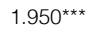 \\
\hline & (166.99) & $(173.12)$ & (183.25) & (191.05) & (190.96) & (180.89) & (187.55) & (182.53) & (186.65) \\
\hline Observaciones & 55596 & 66576 & 66970 & 66174 & 62815 & 57750 & 57345 & 55224 & 55509 \\
\hline$R^{2}$ Ajustado & 0.309 & 0.303 & 0.302 & 0.292 & 0.284 & 0.274 & 0.272 & 0.273 & 0.273 \\
\hline $\mathrm{F}$ & 5277 & 6123 & 6034 & 5722 & 5236 & 4543 & 4581 & 4220 & 4315 \\
\hline
\end{tabular}

Nota: Los cálculos están corregidos por el método de White. ${ }^{*} p<.90 ;{ }^{* *} p<.95 ;{ }^{* * *} p<.99$. Estadístico t entre paréntesis. Fuente: Elaboración propia con base en la ENE (1996-2004) y ENOE (2005-2012). 
Tabla 3. Rendimientos de la educación, 1995-2012: variables instrumentales

\begin{tabular}{|c|c|c|c|c|c|c|c|c|c|}
\hline & 1995 & 1996 & 1997 & 1998 & 1999 & 2000 & 2001 & 2002 & 2003 \\
\hline \multirow[t]{2}{*}{ Escolaridad } & $0.121^{\star \star \star}$ & $0.125^{\star \star \star}$ & $0.124^{\star \star \star}$ & $0.124^{\star \star \star}$ & $0.111^{\star \star *}$ & $0.107^{\star \star *}$ & $0.100^{\star \star *}$ & $0.094^{\star \star \star}$ & $0.094^{\star \star \star}$ \\
\hline & (22.1) & (51.68) & $(38.96)$ & (81.56) & $(45.85)$ & (93.09) & $(91.16)$ & (92.09) & $(90.46)$ \\
\hline \multirow[t]{2}{*}{ Experiencia } & $0.043^{* * *}$ & $0.042^{\star \star \star}$ & $0.040^{\star \star \star}$ & $0.041^{\star \star \star}$ & $0.037^{\star \star \star}$ & $0.035^{\star \star \star}$ & $0.033^{\star \star \star}$ & $0.032^{\star \star \star}$ & $0.033^{\star \star \star}$ \\
\hline & (36.32) & (65.38) & (39.65) & (69.22) & (34.91) & $(64.31)$ & (62.49) & (60.53) & $(57.26)$ \\
\hline \multirow[t]{2}{*}{ Experiencia 2} & $-0.01^{\star \star \star}$ & $-0.00^{\star \star \star}$ & $-0.00^{* * *}$ & $-0.00^{\star \star \star}$ & $-0.00^{\star * *}$ & $-0.00^{\star \star \star}$ & $-0.00^{\star \star \star}$ & $-0.00^{\star \star \star}$ & $-0.00^{\star \star \star}$ \\
\hline & $(-20.32)$ & $(-31.36)$ & $(-17.92)$ & $(-32.18)$ & $(-16.91)$ & $(-30.80)$ & $(-31.45)$ & $(-30.94)$ & $(-29.69)$ \\
\hline \multirow[t]{2}{*}{ Sexo } & $0.072^{\star \star \star}$ & $0.089^{\star \star \star}$ & $0.104^{\star \star \star}$ & $0.109^{\star \star \star}$ & $0.110^{\star \star \star}$ & $0.154^{\star \star \star}$ & $0.170^{\star \star \star}$ & $0.160^{\star \star \star}$ & $0.163^{\star \star \star}$ \\
\hline & $(8.84)$ & (17.94) & (12.92) & $(23.61)$ & (13.39) & $(36.67)$ & $(41.55)$ & (39.04) & $(37.06)$ \\
\hline \multirow[t]{2}{*}{ Constante } & $0.108^{* * *}$ & $0.167^{\star \star \star}$ & $0.393^{\star \star \star}$ & $0.522^{\star \star \star}$ & $0.848^{* * *}$ & $1.105^{\star \star *}$ & $1.304^{\star \star *}$ & $1.441^{\star \star \star}$ & $1.483^{\star \star \star}$ \\
\hline & $(4.73)$ & $(5.85)$ & $(10.72)$ & (29.13) & $(30.10)$ & $(81.85)$ & (99.37) & (114.55) & $(113.87)$ \\
\hline Observaciones & 27566 & 70436 & 24879 & 77310 & 22649 & 91418 & 89459 & 86447 & 76925 \\
\hline$R^{2}$ Ajustado & 0.236 & 0.224 & 0.220 & 0.261 & 0.275 & 0.292 & 0.306 & 0.320 & 0.344 \\
\hline $\begin{array}{l}\mathrm{R}^{2} \text { Parcial } \\
\text { instrumento }\end{array}$ & 0.022 & 0.033 & 0.04 & 0.07 & 0.09 & 0.118 & 0.135 & 0.157 & 0.182 \\
\hline $\mathrm{F}$ instrumento & 625.5 & 2394 & 1264 & 6364 & 2416 & 12229 & 14035 & 16094 & 17204 \\
\hline $\begin{array}{l}\text { excluido } \\
\text { Hausman }\end{array}$ & 11.13 & 104 & & col & 48.58 & 204.7 & 146.4 & 123.1 & 135 \\
\hline
\end{tabular}

Tabla 3. Continuación

\begin{tabular}{|c|c|c|c|c|c|c|c|c|c|}
\hline & 2004 & 2005 & 2006 & 2007 & 2008 & 2009 & 2010 & 2011 & 2012 \\
\hline \multirow[t]{2}{*}{ Escolaridad } & $0.093^{\star \star \star *}$ & $0.100^{\star \star \star}$ & $0.096^{\star \star \star}$ & $0.096^{\star \star \star}$ & $0.091^{\star \star *}$ & $0.088^{* \star *}$ & $0.087^{\star \star *}$ & $0.086^{\star \star \star}$ & $0.087^{\star \star \star}$ \\
\hline & $(80.21)$ & $(71.20)$ & (67.36) & $(65.28)$ & (61.18) & $(56.47)$ & $(54.43)$ & (51.38) & $(49.64)$ \\
\hline \multirow[t]{2}{*}{ Experiencia } & $0.033^{\star \star *}$ & $0.031^{\star * \star}$ & $0.030^{\star \star \star}$ & $0.028^{\star \star \star}$ & $0.028^{* * *}$ & $0.027^{\star \star *}$ & $0.025^{\star \star *}$ & $0.026^{\star \star \star}$ & $0.025^{\star \star \star}$ \\
\hline & $(48.76)$ & (53.28) & (51.08) & $(47.86)$ & $(47.04)$ & $(43.72)$ & $(41.57)$ & (42.62) & $(40.65)$ \\
\hline \multirow[t]{2}{*}{ Experiencia 2} & $-0.00^{\star \star \star}$ & $-0.00^{* * *}$ & $-0.00^{\star * *}$ & $-0.00^{\star \star \star}$ & $-0.00^{\star \star \star}$ & $-0.00^{\star \star \star}$ & $-0.00^{* * *}$ & $-0.00^{\star \star *}$ & $-0.00^{\star \star \star}$ \\
\hline & $(-26.05)$ & $(-25.02)$ & $(-23.82)$ & $(-21.35)$ & $(-22.81)$ & $(-21.66)$ & $(-20.43)$ & $(-22.40)$ & $(-21.18)$ \\
\hline \multirow[t]{2}{*}{ Sexo } & $0.170^{* \star *}$ & $0.193^{\star * *}$ & $0.183^{\star \star \star}$ & $0.181^{\star * \star}$ & $0.188^{* * *}$ & $0.173^{* * *}$ & $0.165^{\star \star *}$ & $0.151^{\star \star \star}$ & $0.155^{\star \star \star}$ \\
\hline & (32.91) & $(41.61)$ & $(40.27)$ & (39.79) & (40.39) & $(35.81)$ & (33.99) & $(30.81)$ & (31.93) \\
\hline \multirow[t]{2}{*}{ Constante } & $1.530^{\star \star *}$ & $1.498^{\star \star \star}$ & $1.633^{\star \star \star}$ & $1.696^{\star \star \star}$ & $1.775^{\star \star \star}$ & $1.819^{\star * \star}$ & $1.879^{\star \star \star}$ & $1.934^{\star \star \star}$ & $2.006^{\star \star *}$ \\
\hline & (104.53) & (88.65) & $(95.60)$ & (94.58) & (96.29) & (92.66) & (93.36) & (93.07) & $(97.10)$ \\
\hline Observaciones & 55596 & 66576 & 66970 & 66174 & 62815 & 57750 & 57345 & 55224 & 55509 \\
\hline$R^{2}$ Ajustado & 0.366 & 0.331 & 0.328 & 0.318 & 0.319 & 0.328 & 0.322 & 0.313 & 0.321 \\
\hline $\begin{array}{l}\mathrm{R}^{2} \text { Parcial } \\
\text { instrumento }\end{array}$ & 0.209 & 0.155 & 0.153 & 0.146 & 0.148 & 0.152 & 0.148 & 0.142 & 0.144 \\
\hline $\mathrm{F}$ instrumento & 14728 & 12227 & 12165 & 11344 & 10986 & 18299 & 10013 & 9145 & 9337 \\
\hline $\begin{array}{l}\text { excluido } \\
\text { Hausman }\end{array}$ & 101.1 & 73.5 & & & & 7.37 & 2.72 & 0.01 & 9.29 \\
\hline
\end{tabular}

Nota: Los cálculos están corregidos por el método de White. . ${ }^{*} \mathrm{p}<.90 ;{ }^{* *} \mathrm{p}<.95 ;{ }^{* * *} \mathrm{p}<.99$. Estadístico t entre paréntesis. Fuente: Elaboración propia con base en la ENE (1996-2004) y ENOE (2005-2012). 
Tabla 4. Resultados de la estimación por niveles educativos, 1995-2012

\begin{tabular}{|c|c|c|c|c|c|c|c|c|c|}
\hline & 1995 & 1996 & 1997 & 1998 & 1999 & 2000 & 2001 & 2002 & 2003 \\
\hline \multirow[t]{2}{*}{ Experiencia } & $0.043^{* * *}$ & $0.044^{* * *+}$ & $0.043^{*+*}$ & $0.044^{* * * *}$ & $0.040^{*+* *}$ & $0.038^{* *+*}$ & $0.037^{*+*}$ & $0.036^{*+* t}$ & $0.036^{2+x+x}$ \\
\hline & (38.79) & (68.63) & $(41.10)$ & (72.08) & $(37.06)$ & (68.52) & (66.08) & $(64.85)$ & (61.53) \\
\hline \multirow[t]{2}{*}{ Experiencia2 } & $-0.01^{* * *}$ & $-0.01^{n+* *}$ & $-0.01^{\star \star * *}$ & $-0.01^{+* *}$ & $-0.01^{*+*}$ & $-0.01^{\text {t**x}}$ & $-0.01^{* * * *}$ & $-0.01^{+*+*}$ & $-0.01^{* * * t}$ \\
\hline & $(-26.66)$ & $(-45.80)$ & $(-26.81)$ & $(-48.51)$ & $(-25.26)$ & $(-47.10)$ & $(-46.00)$ & $(-45.04)$ & $(-43.54)$ \\
\hline \multirow[t]{2}{*}{ Primaria } & & $0.216^{* * *}$ & $0.256^{\text {*n+x }}$ & $0.210^{* n * *}$ & $0.177^{* \star *}$ & $0.196^{* * t}$ & $0.192^{*+*}$ & $0.174^{*+* *}$ & $0.145^{*+*}$ \\
\hline & (12.68) & (15.73) & (11.23) & (15.38) & (6.69) & (14.39) & (14.05) & $(12.70)$ & (10.08) \\
\hline \multirow[t]{2}{*}{ Secundaria } & $0.715^{* * *}$ & $0.591^{* * *}$ & $0.614^{*+*}$ & $0.587^{* * *}$ & $0.526^{*+* *}$ & $0.481^{* * *}$ & $0.449^{*+*}$ & $0.409^{*+4 *}$ & $0.365^{n+x}$ \\
\hline & $(27.28)$ & (41.39) & (25.85) & $(41.44)$ & (19.22) & $(34.40)$ & (31.99) & (29.05) & (24.64) \\
\hline \multirow[t]{2}{*}{ Bachillerato } & $0.959^{* * * *}$ & $0.837^{*+*}$ & $0.888^{*+* x}$ & $0.832^{* * * *}$ & $0.751^{* \ldots+x}$ & $0.704^{* * *}$ & $0.653^{\text {*n+ }}$ & $0.601^{\text {*n* }}$ & $0.554^{* x+x}$ \\
\hline & (34.05) & $(54.61)$ & $(34.76)$ & $(55.23)$ & (26.12) & $(48.13)$ & $(44.70)$ & $(40.94)$ & (35.96) \\
\hline \multirow[t]{2}{*}{ Universidad } & $1.649^{* * *}$ & $1.531^{\text {t*t*}}$ & $1.575^{x+x}$ & $1.534^{m+*}$ & $1.425^{\star * *}$ & $1.374^{*+*}$ & $1.302^{*+*}$ & $1.234^{*+* x}$ & $1.184^{* *+}$ \\
\hline & $(58.42)$ & $(100.14)$ & $(62.04)$ & (101.92) & $(49.47)$ & (92.76) & (87.98) & (83.35) & (75.83) \\
\hline \multirow[t]{2}{*}{ Posgrado } & $2.148^{* *+*}$ & $1.995^{*+*}$ & $2.070^{*+*}$ & $1.967^{* * *}$ & $1.876^{*+*+}$ & $1.884^{*+*}$ & $1.796^{*+*}$ & $1.740^{*+* *}$ & $1.733^{*+x+}$ \\
\hline & $(44.03)$ & $(74.78)$ & (52.79) & (81.52) & $(41.07)$ & (81.39) & $(76.71)$ & (78.18) & (71.94) \\
\hline \multirow[t]{2}{*}{ Constante } & $0.539^{* * * *}$ & $0.771^{* * * *}$ & $0.949^{* * *}$ & $1.134^{* * *}$ & $1.383^{* * * x}$ & $1.661^{* * *}$ & $1.832^{* * *}$ & $1.949^{* * * *}$ & $2.036^{k+1}$ \\
\hline & (20.12) & $(51.45)$ & $(38.16)$ & $(76.86)$ & $(48.95)$ & (115.28) & (126.52) & $(134.20)$ & (133.13) \\
\hline Observaciones & 27566 & 70436 & 24879 & 77310 & 22649 & 91418 & 89459 & 86447 & 76925 \\
\hline$R^{2}$ ajustado & 0.322 & 0.357 & 0.376 & 0.363 & 0.351 & 0.320 & 0.311 & 0.304 & 0.296 \\
\hline $\mathrm{F}$ & 1651 & 4950 & 1973 & 5838 & 1626 & 5476 & 5063 & 4875 & 4052 \\
\hline
\end{tabular}

Tabla 4. Continuación

\begin{tabular}{|c|c|c|c|c|c|c|c|c|c|}
\hline & 2004 & 2005 & 2006 & 2007 & 2008 & 2009 & 2010 & 2011 & 2012 \\
\hline \multirow[t]{2}{*}{ Experiencia } & $0.036^{* *+*}$ & $0.036^{* * *}$ & $0.035^{* * *}$ & $0.033^{\text {t** }}$ & $0.033^{* * *}$ & $0.032^{*+* t}$ & $0.031^{* * * *}$ & $0.032^{* * *}$ & $0.031^{* n *}$ \\
\hline & $(52.14)$ & (61.43) & (59.59) & (57.15) & (56.04) & (52.58) & (50.49) & (51.33) & (49.92) \\
\hline \multirow[t]{2}{*}{ Experiencia2 } & $-0.01^{k+* *}$ & $-0.01^{* * *}$ & $-0.01^{* * *}$ & $-0.01^{* * * *}$ & $-0.01^{\star * * *}$ & $-0.01^{*+* *}$ & $-0.01^{* * *}$ & $-0.01^{+* * *}$ & $-0.01^{* * *}$ \\
\hline & $(-37.31)$ & $(-43.65)$ & $(-41.06)$ & $(-39.56)$ & $(-39.48)$ & $(-37.62)$ & $(-35.57)$ & $(-37.24)$ & $(-35.34)$ \\
\hline \multirow[t]{2}{*}{ Primaria } & $0.143^{* * *}$ & $0.119^{* * *}$ & $0.126^{*+*}$ & $0.126^{* * *}$ & $0.109^{* * *}$ & $0.068^{* \star *}$ & $0.097^{*+*}$ & $0.109^{* * *}$ & $0.075^{\star \star *}$ \\
\hline & $(8.02)$ & $(7.46)$ & $(7.38)$ & $(7.02)$ & (6.03) & $(3.49)$ & $(5.10)$ & $(5.82)$ & (3.94) \\
\hline \multirow[t]{2}{*}{ Secundaria } & $0.378^{* * *}$ & $0.274^{* * * *}$ & $0.283^{*+* x}$ & $0.268^{\operatorname{t*x} x}$ & $0.245^{* * *}$ & $0.196^{* n+*}$ & $0.228^{* * *}$ & $0.227^{\star * *}$ & $0.198^{\mathrm{ck*}}$ \\
\hline & (20.76) & (16.72) & (16.15) & $(14.70)$ & (13.24) & $(9.84)$ & (11.83) & (11.98) & (10.26) \\
\hline \multirow[t]{2}{*}{ Bachillerato } & $0.554^{* \ldots+}$ & $0.503^{* * *}$ & $0.497^{*+* *}$ & $0.481^{\text {t** }}$ & $0.455^{* * *}$ & $0.398^{* *+}$ & $0.431^{* * *+}$ & $0.434^{* * *}$ & $0.392^{\ldots+*}$ \\
\hline & $(29.27)$ & $(30.27)$ & (28.02) & (26.01) & (24.36) & (19.81) & (22.12) & (22.62) & (20.03) \\
\hline \multirow[t]{2}{*}{ Universidad } & $1.180^{*+* t}$ & $1.053^{* * * *}$ & $1.055^{* * *}$ & $1.022^{* * * *}$ & $0.989^{* * *}$ & $0.929^{*+*}$ & $0.950^{* * * *}$ & $0.946^{* * *}$ & $0.910^{* * * *}$ \\
\hline & $(61.48)$ & (62.19) & $(58.51)$ & (54.56) & (52.06) & $(45.53)$ & (48.03) & (48.34) & $(45.73)$ \\
\hline \multirow[t]{2}{*}{ Posgrado } & $1.728^{* * *+}$ & $1.576^{* * * *}$ & $1.581^{\text {t** }}$ & $1.508^{n+*}$ & $1.476^{* * *}$ & $1.399^{* * *}$ & $1.468^{* * *}$ & $1.446^{\text {t*t+ }}$ & $1.437^{k \star * x}$ \\
\hline & (62.94) & $(65.27)$ & $(63.40)$ & $(59.70)$ & (57.97) & (51.68) & (55.93) & (54.13) & (53.93) \\
\hline \multirow[t]{2}{*}{ Constante } & $2.075^{* * *}$ & $2.173^{* * * *}$ & $2.242^{4+* x}$ & $2.327^{\star \star * *}$ & $2.381^{x * *}$ & $2.435^{\text {t*t }}$ & $2.444^{* * *}$ & $2.458^{* * *}$ & $2.517^{* * *}$ \\
\hline & (110.10) & (129.88) & (126.40) & (125.64) & (126.81) & (120.59) & (124.42) & (126.96) & (127.81) \\
\hline Observaciones & 55596 & 66576 & 66970 & 66174 & 62815 & 57750 & 57345 & 55224 & 55509 \\
\hline$R^{2}$ ajustado & 0.292 & 0.299 & 0.304 & 0.290 & 0.284 & 0.277 & 0.276 & 0.276 & 0.277 \\
\hline $\mathrm{F}$ & 2899 & 3538 & 3613 & 3345 & 3139 & 2722 & 2755 & 2516 & 2567 \\
\hline
\end{tabular}

Nota: Ecuación estimada: $\operatorname{Ln} W=\beta 0+\beta 1 X+\beta 2 X 2+a 1 D n 1+a 2 D n 2+a 3 D n 3+a 4 D n 4+a 5 D n 5+\mu .{ }^{*} p<.90 ;{ }^{* *} p<.95 ;{ }^{* * *}$ $p<.99$. Estadístico t entre paréntesis.

Fuente: Elaboración propia con base en la ENE (1995-2004) y ENOE (2004-2005). 
Tabla 5. Rendimientos absolutos y marginales por nivel educativo, 1995-2012

\begin{tabular}{|c|c|c|c|c|c|c|}
\hline Años & $\begin{array}{l}\text { Rendimiento } \\
\text { medio }\end{array}$ & Primaria & Secundaria & Bachillerato & Universidad & Posgrado \\
\hline \multicolumn{2}{|c|}{ Adicionales de estudio } & \multicolumn{5}{|c|}{ Absolutos } \\
\hline & & (6) & (9) & (12) & $(16)$ & $(18)$ \\
\hline 1995 & 10.30 & 4.38 & 6.94 & 7.99 & 10.31 & 11.93 \\
\hline 1996 & 10.10 & 3.60 & 6.57 & 6.98 & 9.57 & 11.08 \\
\hline 1997 & 10.20 & 4.27 & 6.82 & 7.40 & 9.84 & 11.50 \\
\hline 1998 & 10.10 & 3.50 & 6.52 & 6.93 & 9.59 & 10.93 \\
\hline 1999 & 9.50 & 2.95 & 5.84 & 6.26 & 8.91 & 10.42 \\
\hline 2000 & 9.20 & 3.27 & 5.34 & 5.87 & 8.59 & 10.47 \\
\hline 2001 & 8.70 & 3.20 & 4.99 & 5.44 & 8.14 & 9.98 \\
\hline 2002 & 8.40 & 2.90 & 4.54 & 5.01 & 7.71 & 9.67 \\
\hline 2003 & 8.30 & 2.42 & 4.06 & 4.62 & 7.40 & 9.63 \\
\hline 2004 & 8.30 & 2.38 & 4.20 & 4.62 & 7.38 & 9.60 \\
\hline 2005 & 8.90 & 1.98 & 3.04 & 4.19 & 6.58 & 9.27 \\
\hline 2006 & 8.90 & 2.10 & 3.14 & 4.14 & 6.59 & 9.30 \\
\hline 2007 & 8.70 & 2.10 & 2.98 & 4.01 & 6.39 & 9.43 \\
\hline 2008 & 8.50 & 1.82 & 2.72 & 3.79 & 6.18 & 9.23 \\
\hline 2009 & 8.40 & 1.13 & 2.18 & 3.32 & 5.81 & 8.74 \\
\hline 2010 & 8.40 & 1.62 & 2.53 & 3.59 & 5.94 & 9.18 \\
\hline 2011 & 8.40 & 1.82 & 2.52 & 3.62 & 5.91 & 9.04 \\
\hline \multirow[t]{3}{*}{2012} & 8.50 & 1.25 & 2.20 & 3.27 & 5.69 & 8.98 \\
\hline & & \multicolumn{5}{|c|}{ Marginales } \\
\hline & & (6) & (3) & (3) & (4) & (3) \\
\hline 1995 & 10.30 & 5.38 & 6.53 & 8.13 & 13.80 & 17.67 \\
\hline 1996 & 10.10 & 3.60 & 6.25 & 8.20 & 13.88 & 18.22 \\
\hline 1997 & 10.20 & 4.27 & 5.97 & 8.56 & 13.74 & 18.33 \\
\hline 1998 & 10.10 & 3.50 & 6.28 & 8.17 & 14.04 & 18.96 \\
\hline 1999 & 9.50 & 2.95 & 5.82 & 7.50 & 13.48 & 18.22 \\
\hline 2000 & 9.20 & 3.27 & 5.70 & 7.43 & 13.40 & 17.87 \\
\hline 2001 & 8.70 & 3.20 & 5.14 & 6.80 & 12.98 & 17.57 \\
\hline 2002 & 8.40 & 2.90 & 4.70 & 6.40 & 12.66 & 18.27 \\
\hline 2003 & 8.30 & 2.42 & 4.40 & 6.30 & 12.60 & 19.00 \\
\hline 2004 & 8.30 & 2.38 & 4.70 & 7.04 & 12.52 & 18.43 \\
\hline 2005 & 8.90 & 1.98 & 5.17 & 7.63 & 12.22 & 18.77 \\
\hline 2006 & 8.90 & 2.10 & 5.23 & 7.13 & 12.40 & 18.03 \\
\hline 2007 & 8.70 & 2.10 & 4.73 & 7.10 & 12.02 & 17.39 \\
\hline 2008 & 8.50 & 1.82 & 4.53 & 7.00 & 11.87 & 17.75 \\
\hline 2009 & 8.40 & 1.13 & 4.27 & 6.73 & 11.80 & 17.38 \\
\hline 2010 & 8.40 & 1.62 & 4.37 & 6.77 & 11.53 & 17.47 \\
\hline 2011 & 8.40 & 1.82 & 3.93 & 6.90 & 11.38 & 17.37 \\
\hline 2012 & 8.50 & 1.25 & 4.10 & 6.47 & 11.51 & 18.93 \\
\hline
\end{tabular}

Nota: Los rendimientos absolutos se obtienen respecto de la categoría sin estudios. Los rendimientos marginales se obtienen respecto de los del nivel educativo anterior.

Fuente: Elaboración propia con base en los resultados de la tabla 4.

E. M. Villarreal Peralta | Endogeneidad de los rendimientos educativos en México | Perfiles Latinoamericanos Flacso México | Perfiles Latinoamericanos, 26(51) Flacso México | pp. 265-299 


\section{Referencias}

Acemoglu, D. \& Angrist, J. (2000). How Large are the Social Returns to Education: Evidence from Compulsory Schooling Laws. NBER Working Paper W7444. Cambridge: National Bureau of Economic Research.

Airola, J. \& Juhn, C. (2005). Wage Inequality in Post-Reform Mexico. IzA Discussion Papers 1525. Bonn: Institute for the Study of Labour.

Altonji, J. (1993). The Demand for and Return to Education when Education Outcomes are Uncertain. Journal of Labor Economics, 11(1), 48-83.

Angrist, J. \& Imbens, G. (1995). Two-Stage Least Squares Estimation of Average Causal Effects in Models with Variable Treatment Effect. Journal of the American Statistical Association, 90(430), 431-442.

Angrist, J. \& Krueguer, A. (1991). Does compulsory school attendance affect schooling and earnings. Quarterly Journal of Economics, 106(4), 974-1014.

Ashenfelter, O. \& Krueger, A. (1994). Estimates of the Economic Return to Schooling. The American Economic Review, 84(5), 1157-1173.

Barceinas, F. (2003). Endogeneidad y rendimientos de la educación. Estudios Económicos, 18(1), $79-131$

Barceinas, F. (2002). Rendimientos privados y sociales de la educación en México. Economía Mexicana, Nueva Época, 11(2), 333-390.

Barceinas, F. (1999). Función de ingresos y rendimiento de la educación en México. Estudios Económicos, 14(1), 87-127.

Barceinas Paredes, F. \& Raymond-Bara, J. L. (2005). Convergencia regional y capital humano en México, de los ańos 80 al 2000. Estudios Económicos, 20(2), 263-305.

Barceinas Paredes, F. \& Raymond-Bara, J. L. (2003). Hipótesis de señalización versus capital humano. El caso de México. El Trimestre Económico, LXX-1(277),167-194.

Becker, G. (1964). Human Capital: A Theoretical and Empirical Analysis, with Special Reference to Education. Nueva York: Columbia University Press.

Becker, G. (1962). Investment in Human Capital: A Theoretical Analysis. The Journal of Political Economy, 66(2), 281-302. 
Binelli C. \& Rubio, M. (2012). The Returns to Private Education: Evidence from Mexico. Institute for Fiscal Studies, Working Papers W12/08. Londres: Centre for the Evaluation of Development Policies.

Bosch, M., \& Manacorda, M. (2010). Minimum Wages and Earnings Inequality in Urban Mexico. American Economic Journal: Applied Microeconomics, 2(4), 128-149.

Bound, J., Jaeger, D. \& Baker, R. (1995). Problems with Instrumental Variables Estimation when the Correlation Between the Instruments and the Exogenous Explanatory Variables is Weak. Journal of the American Statistical Association, 90(430), 443-450.

Bracho, T. \& Zamudio, A. (1994). Los rendimientos económicos de la escolaridad en México 1989. Economia Mexicana, $\operatorname{III}(2), 345-377$.

CAIInno (2016). Panorama General de la Educación en México. Centro de Análisis para la Investigación en Innovación (CAIINNo). Recuperado el 6 de octubre de 2015, de http://www .caiinno.org/temas/ciencia-y-tecnologia/educacion-en-mexico/

Campos, R. M. (2010). Why did Wage Inequality Decrease in Mexico After Nafta? Serie Documentos de Trabajo 15. México: El Colegio de México.

Campos, R. M., Esquivel, G. \& Lustig, N. (2012). The Rise and Fall of Income Inequality in Mexico, 1989-2010. Society for the Study of Economic Inequality (ECINEQ). Working Paper Series 267.

Cantillón, R. (1950). Ensayo sobre la naturaleza del comercio en general. México: Fondo de Cultura Económica.

Cañonero G. \& Werner, A. (2002). Salarios relativos y liberación del comercio en México. El Trimestre Económico, (273), 123-142.

Card, D. (2000). Estimating the Return to Schooling: Progress on Some Persistent Econometric Problems. NBER Working Paper 7769. Cambridge: National Bureau of Economic Research.

Card, D. (1999). The Causal Effect of Education on Earnings. En Ashenfelter \& Card, D. (Eds.). Handbook of Labor Economics, 3A. Amsterdam: Elsevier.

Card, D. (1993). Using Geographic Variation in College Proximity to Estimate the Return to Schooling. NBER Working Paper 4483, Cambridge: National Bureau of Economic Research.

Card, D. \& Krueger. A. (1992). Does School Quality Matter? Returns to Education and the Characteristics of Public Schools in the United States. Journal of Political Economy, (100), 1-40. 
Carnoy, M. (1967). Earnings and Schooling in Mexico. Economic Development and Cultural Change, 15(4), 408-418.

Chiquiar, D. (2004). Why Mexico's Regional Income Convergence Broke Down. Journal of Development Economics, 77, 257-275.

Chiquiar, D. \& Hanson, G. (2002). International Migration, Self-Selection, and the Distribution of Wage: Evidence from Mexico and United States. NBER Working Paper 9242.

Cohn, E. \& Hughes, W. (1994). A Benefit-Cost Analysis of Investment in College Education in the United States: 1969-1985. Economics of Education Review, 13(2), 109-123.

Comisión Económica para América Latina y el Caribe (CEPAL). (2016). Productividad y brechas estructurales en México. México: CEPAL.

Cortez, W. (2001). What is Behind Increasing Wage Inequality in Mexico? World Development, (29), 1905-1922.

Cragg, M. \& Epelbaum, M. (1996). Why Has Wage Dispersion Grown in México? Is it Incidence of Reforms or the Growing Demand for Skills? Journal of Development Economics, (51), 99-116.

De Ferranti, D., Perry, G., Ferreira, F. \& Walton, M. (2003). Inequality in Latin America Breaking with History? Washington: The World Bank.

INEGI (1995-2004). Encuesta Nacional de Empleo (ENE) (1995-2004)Instituto Nacional de Estadística y Geografía (INEGI). Recuperado el 9 de octubre de 2015, de http://www.beta.inegi .org.mx/proyectos/enchogares/historicas/ene/

INEGI (2005-2012). Encuesta Nacional de Ocupación y Empleo (ENOE). Instituto Nacional de Estadística y Geografía (INEGI). Recuperado el 09 de octubre de 2015, de http://www.beta .inegi.org.mx/proyectos/enchogares/historicas/enoe/default.html

Esquivel, G., Lustig, N. \& Scott, J. (2010). A Decade of Falling Inequality in Mexico: Market Forces or State Action? En Lopez-Calva, L. F. \& Lustig, N. (Eds.). Declining Inequality in Latin America: A Decade of Progress? Washington: Brookings Institution/undP.

Esquivel, G. \& Rodríguez-López, J. A. (2003). Technology, Trade and Wage Inequality. Journal of Development Economics, 72(2), 543-65.

Fairris, D. (2003). Unions and Wage Inequality in Mexico. Industrial and Labour Relations Review, 56(3), 481-97. 
Fairris, D., Popli, G. \& Zepeda, E. (2008). Minimum Wages and the Wage Structure in Mexico. Review of Social Economy, 66(2), 181-208.

Feliciano, Z. (2001). Workers and Trade Liberalization. The Impact of Trade Reform. The Case of Mexico. Industrial and Labour Relations Review, 55(1), 95-115.

Ghiara R. \& Zepeda, E. (2004). México: Las crecientes diferencias salariales por tipo de industria. Comercio Exterior, 54(1), 48-60.

Ginther, D. (2000). Alternative Estimates of the Effect of Schooling on Earnings. The Review of Economics and Statistics, 82(1), 103-116.

Gómez, L. \& Psacharopoulos, G. (1990). Earnings and Education in Ecuador: Evidence from the 1987 Household Survey. Economics of Education Review, 9(3), 219-227.

Griliches, Z. (1977). Estimating the Return to Schooling: Some Econometric Problems. Econometrica, 45(1), 1-22.

Griliches, Z. \& Jorgenson, D. W. (1966). Sources of Measured Productivity Change: Capital Input. American Economic Review, 61(2), 50-61.

Hanoch, G. (1967). An Economic Analysis of Earnings and Schooling. Journal of Human Resources, 2(3), 310-329.

Hansen, W. L. (1963). Total and Private Rates of Return to Investment in Schooling. Journal of Political Economy, 71, 128-140.

Hanson, G. (2003). What has Happened to Wages in Mexico since NAFTA? Implications for Hemispheric Free Trade. NBER Working Paper 9563. Cambridge: National Bureau of Economic Research.

Hanson, G. \& Harrison, H. (1999). Trade Liberalization and Wage Inequality. Industrial and Labour Relations Review, 52(2), 271-88.

Harberger, A. \& Peón, G. (2012). Estimating Private Returns to Education in Mexico. Latin American Journal of Economics, 49(1), 1-35.

Harmon, C. \& Walker, I. (2001). The Returns to Education-A Review of Evidence, Issues and Deficiencies in the Literature. Research Report 254. Londres: Department for Education and Employment.

Harmon, C. \& Walker, I. (1995). Economic Return to Schooling for the UK. American Economic Review, 85(5), 1278-1286. 
Heckman, J. (1997). Instrumental Variables: A Study of Implicit Behavioral Assumptions Used In Making Program Evaluations. Journal of Human Resources, 32(3), 441-462.

Huesca, L. (2004). La rentabilidad de la escolaridad en los hogares asalariados de México durante 1984-2000. Problemas del Desarrollo, 35(138), 126-154.

Lachler, U. (1998). Education and Earnings Inequality in México. Working Paper 1949. World Bank Policy Research.

López-Acevedo, G. (2006). Mexico: Two Decades of the Evolution of Education and Inequality. Working Paper 3919. World Bank Policy Research.

López-Acevedo, G. (2004). México: Evolution of Earnings inequality and Rates of Returns to Education (1988-2002). Estudios Económicos, 19(2), 211-284.

Lopez-Calva, L. F. \& Lustig, N. (2009). The Recent Decline of Inequality in Latin America: Argentina, Brazil, Mexico and Peru. Society for the Study of Economic Inequality. ECINEQ Working Paper Series 140.

Lustig, N., Lopez-Calva, L. F. \& Ortiz-Juárez, E. (2013). Declining Inequality in Latin America in the 2000s: the Cases of Argentina, Brazil, and Mexico. World Development, (44), 129-141.

McMahon, W. W. (1991). Relative Returns to Human and Physical Capital in the U. S. and Efficient Investment Strategies. Economics of Education Review, (10), 283-296.

Manacorda, M., Sánchez-Páramo, C. \& Schady, N. (2010). Changes in Returns to Education in Latin America: The Role of Demand and Supply of Skills. Industrial and Labor Relations Review, (63), 307-326.

Mehta, A. \& Villarreal, H. (2008). Why do Diplomas Pay? An Expanded Mincerian Framework Applied to Mexico. Applied Economics, 40(24), 3127-3144.

Meza, L. (2005). Mercados laborales locales y desigualdad salarial en México. El Trimestre Económico, (285), 133-178.

Meza, L. (1999). Cambios en la estructura salarial de México en el periodo 1988-1993 y el aumento en el rendimiento de la educación superior. El Trimestre Económico, (262), 189-226.

Mincer, J. (1974). Schooling, Experience and Earnings. Nueva York: National Bureau of Economic Research/Columbia University Press.

Mincer, J. (1962). On the Job Training: Cost, Returns and Some Implications. Journal of Political Economy, 70(5), 50-79. 
Morales, E. (2011). Los rendimientos de la educación en México. Documento de Investigación 2011/07. México: Banco de México.

Ordaz, J. (2007). México: Capital humano e ingresos. Retornos a la educación, 1994-2005. MéxiCO: CEPAL.

Psacharopoulos, G. (1994). Returns to Investment in Education: A Global Update. World Development, 22(9), 1325-1343.

Psacharopoulos, G. \& Ng, Y. (1992). Earnings and education in Latin America: Assessing Priorities for Schooling Investment. Education Economics, 2(2).

Psacharopoulos, G. \& Patriños, H. (2002). Returns to Investment in Education: A Further Update. Policy Research Working Paper 2881. The World Bank.

Psacharopoulos, G., Vélez, E., Panagides, A. \& Yang, H. (1996). Returns to Education During Economic Boom and Recession: Mexico, 1984, 1989 and 1992. Education Economics, 4(3), 219-230.

Revenga, A. (1997). Employment and Wage Effects of Trade Liberalization: The Case of Mexican Manufacturing. Journal of Labor Economics, 15(3), 20-43.

Ríos, J. G. (2005). La educación, las remuneraciones y los salarios en México. Comercio Exterior, 55(5), 402-417.

Robertson, R. (2007). Trade and Wages: Two Puzzles from Mexico. The World Economy, 30(9), 1378-1398.

Robertson, R. (2004). Relative Prices and Wage Inequality: Evidences from Mexico. Journal of International Economics, (64), 387-409.

Rodríguez-Oreggia, E. (2005). Institutions, Geography and the Regional Development of Returns to Schooling in Mexico. Research Institute for Sustainable Development and Social Equality (IIDSES) Working Paper 8. México: Universidad Iberoamericana.

Rojas, M., Angulo, H. \& Velázquez, I. (2000). Rentabilidad de la inversión en capital humano en México, Economía Mexicana, 9(2), 113-142.

Salas, M. (2002). Estimación de la rentabilidad de la inversión en educación universitaria de ciclo largo. Estadistica Española, (149), 89-112.

Salas, M. (2004). Rendimientos privados de las inversiones en educación superior a partir de ecuaciones de ingresos. Hacienda Pública Española, 169(2), 87-117, 
Sapelli, C. (2009). Ecuaciones de Mincer y las Tasas de Retorno a la educación en Chile: 1990-1998. Documento de Trabajo 254. Instituto de Economía, Pontificia Universidad Católica de Chile.

Sariñana, J. (2002). Rendimiento de la escolaridad en México: una aplicación del método de variables instrumentales para 1998. Gaceta de Economía, 7(14), 85-127.

Schultz, T. (1961). Investment in Human Capital. American Economic Review, 51(1), 1-17.

SEP (2015). Reporte de Indicadores Educativos, Dirección General de Planeación y Estadística Educativa de la Secretaría de Educación Pública. Recuperado el 9 de octubre de 2015, de http://www.snie.sep.gob.mx/indicadores_pronosticos.html

Serdán, A. (2012). Panorama del Gasto Educativo en Mexico 2012, Coalición Ciudadana por la Educación. Recuperado el 9 de octubre de 2015, de http://www.culturadelalegalidad .org.mx/recursos/Contenidos/Estadsticas/documentos/Panorama\%20del\%20gasto $\% 20$ educativo\%202012.pdf

Singh R. \& Santiago, M. (1997). Farm Earnings, Educational Attainment, and Role of Public Policy: Some Evidence from Mexico. World Development, 25(12), 2143-2154.

Smith, A. (1776). An Inquiry into the Nature and Causes of the Wealth of Nations. Londres: W. Strahan \& T. Cadell.

Tello, C., Ramos, R. \& Artís, M. (2012). Changes in Wage Structure in Mexico Going Beyond the in Mean: An Analysis of Differences Distribution, 1987-2008. IZA Discussion Paper Series 6576.

United Nations Development Program (UNDP). (2004). Human Development Report. Nueva York: UNDP.

United Nations Development Program (UNDP). (2005). Human Development Report. Nueva York: UNDP.

United Nations Development Program (UNDP). (2006). Human Development Report. Nueva York: UNDP.

United Nations Development Program (UNDP). (2007). Human Development Report. Nueva York: UNDP.

Urciaga, J. (2004). Los impactos del territorio sobre los salarios: una aproximación empírica para México. Prospectiva Económica, 4(1), 135-156. 
Urciaga, J. (2002). Los rendimientos privados de la escolaridad formal en México. Comercio exterior, 52(4), 325-330.

Urciaga, J. \& Almendarez, M. (2006). Determinación de los salarios y rendimientos de la escolaridad en la región Mar de Cortés. Revista de la Educación Superior, 35(2), 37-53.

Urciaga, J. \&. Vukasinak, T. (2002). Salarios, escolaridad y rentabilidad privada de la educación en México. Evidencia con datos de presupuestos familiares de 2000. Prospectiva Económica, 1(1), 151-171.

Villarreal, E. M. (2008). Evolución histórica de los rendimientos educativos en México. Estudios Sociales, 16(32), 59-79.

Zepeda, E. \& Ghiara, R. (1999). Determinación del salario y capital humano en México: 19871993. Economia, Sociedady Territorio, 2(5), 67-116.

Recibo el 25 de noviembre de 2015. Aceptado el 17 de enero de 2017. 